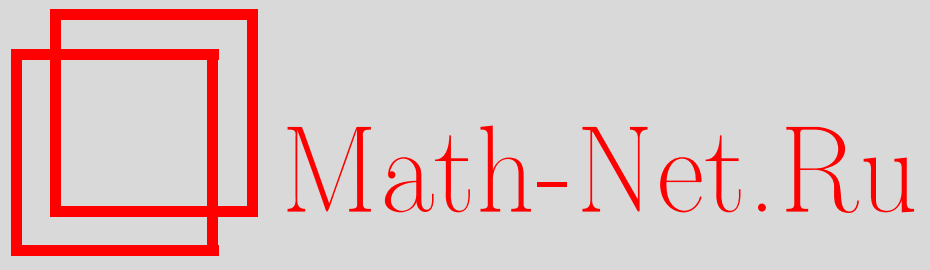

А. В. Кряжимский, Апостериорная интеграция вероятностей. Элементарная теория, Теория вероятн. и ее примен., 2015, том 60, выпуск 1, 45-79

DOI: https://doi.org/10.4213/tvp4605

Использование Общероссийского математического портала Math-Net.Ru подразумевает, что вы прочитали и согласны с пользовательским соглашением http: //www . mathnet.ru/rus/agreement

Параметры загрузки:

IP : 54.224 .135 .184

26 апреля 2023 г., 12:55:29

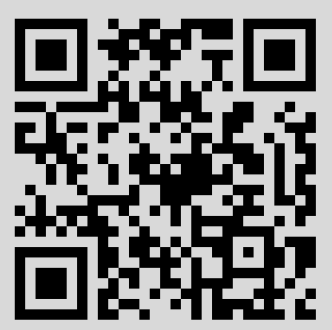




\section{АПОСТЕРИОРНАЯ ИНТЕГРАЦИЯ ВЕРОЯТНОСТЕЙ. ЭЛЕМЕНТАРНАЯ ТЕОРИЯ ${ }^{1)}$}

Предлагается подход к апостериорной интеграции вероятностных распределений, служащих независимыми априорными моделями наблюдаемого элементарного события из заданного конечного набора элементарных событий. Под апостериорной интеграцией понимается уточнение сведений, доставляемых априорными вероятностями. В основу подхода положено понятие апостериорного события в произведении вероятностных пространств, отвечающих априорным вероятностям. Условная вероятность на пространствепроизведении, задаваемая апостериорным событием, естественным образом определяет вероятность на множестве исходных элементарных событий; последняя принимается за результат апостериорной интеграции априорных моделей. Указываются условия, при которых интеграция повышает информативность априорных вероятностей; исследуются алгебраические свойства интеграции как бинарной операции на множестве вероятностей; рассматривается вопрос об интегральной сходимости бесконечных последовательностей вероятностей.

Kлючевые слова и фразы: непротиворечивые методы наблюдения, max-мера сосредоточенности, max-совместимость, маргинальная совместимость, max-концентратор, интеграционная сходимость, интеграционная сосредоточенность.

Введение. При изучении сложных, плохо наблюдаемых систем, прежде всего социально-экономических и систем окружающей среды, часто требуется сопоставление данных, получаемых из альтернативных источников. Такие данные, как правило, плохо согласуются друг с другом. В типичных случаях данные представляются в виде вероятностных распределений, отражающих помехи наблюдения либо моделирования (см., например, [10]). В конкретных прикладных исследованиях для синтеза (интеграции) рассогласованных распределений привлекают те или иные специфические (известные либо допускаемые) свойства исследуемых объектов (см., например, [6]). Универсальные методики по интеграции рассогласованных априорных распределений в практических

\footnotetext{
*Математический институт им. В.А. Стеклова РАН, Москва, Россия; Международный институт прикладного системного анализа, Лаксенбург, Австрия.

1) Работа выполнена при поддержке РФФИ (гранты № 13-01-00685, 13-01-12446офи-м2) и DG Research COMPLEX, Project 308601 (www.complex.ac.uk).
} 
исследованиях концентрируются вокруг идеи взятия их выпуклых комбинаций с коэффициентами, выбираемыми на основе информации о надежности источников этих распределений (см., например, [7], [8], [10]). Такие методики носят в целом эмпирический характер, специалисты признают необходимость разработки соответствующих формализованных подходов (см., например, [5]). Для случаев, когда информация о надежности источников априорных распределений отсутствует, дефицит методик их интеграции является еще более наглядным.

В данной работе, мотивированной обозначенными выше вопросами обработки результатов наблюдений сложных социально-экономических систем и систем окружающей среды, предлагается унифицированный подход к интеграции априорных вероятностных распределений, доставляемых независимыми источниками - «методами наблюдения» при отсутствии какой-либо приоритизации этих источников по критерию надежности. Предполагается, что некоторый детерминированный элемент - значение какого-либо показателя исследуемой социальноэкономической системы или системы окружающей среды - наблюдается несколькими альтернативными методами и результат наблюдения, доставляемый каждым методом, представляет собой вероятностное распределение на множестве всех допустимых элементов (элементарных событий). Мы называем эти вероятностные распределения или, кратко, вероятности априорными. Задача состоит в том, чтобы сконструировать апостериорную вероятность, доставляющую уточненную информацию о наблюдаемом допустимом элементе посредством синтеза данных, доставляемых априорными вероятностями.

В известном смысле эта задача близка к задаче об оценивании неизвестного параметра вероятностного распределения по результатам наблюдения за исходами эксперимента (см. [1, гл. I, $\S 7$, с. 97]). Однако в рассматриваемом случае допустимые элементы не служат параметрами априорных распределений, а для идентификации наблюдаемого допустимого элемента используется не набор эмпирических результатов наблюдения, а семейство альтернативных априорных распределений. Чтобы подчеркнуть своеобразие рассматриваемого случая, можно считать, что априорные распределения суть эмпирические частоты, полученные в результате многократных экспериментов, и, стало быть, неотличимы от вероятностных распределений, характеризующих ошибки соответствующих методов наблюдения.

Множества вероятностных распределений исследуются в литературе с разных точек зрения. Так, теория статистических решений Вальда [11] ориентируется на оптимизацию решений при неопределенном распределении «состояний природы»; в теории сравнения экспериментов (см. [2], [3], [9]) множества вероятностных распределений служат моделями экспериментов, подлежащих сравнению по критерию инфор- 
мативности; ряд исследований посвящен анализу статистических данных, генерируемых различными источниками (см. [4]). В настоящей работе множества вероятностных распределений выступают в качестве материала для синтеза интегральной информации о наблюдаемом элементе.

Предлагаемый подход основан на понятии апостериорного события в произведении вероятностных пространств, соответствующих априорным вероятностям. Определение апостериорного события основано на том факте, что все априорные вероятности являются описаниями одного и того же детерминированного элемента - наблюдаемого элементарного события; следовательно, элементарное событие в пространствепроизведении может быть квалифицировано как апостериорно допустимое лишь тогда, когда все его компоненты идентичны. Совокупность всех апостериорно допустимых элементарных событий составляет апостериорное событие - «диагональ» пространства-произведения. Определенная на пространстве-произведении условная вероятность при условии апостериорного события сконцентрирована на последнем и естественным образом определяет вероятность на множестве исходных элементарных событий; последняя вероятность принимается за результат апостериорной интеграции априорных вероятностей.

Для простоты здесь рассматривается случай конечного множества допустимых элементарных событий. Раздел 1 содержит основные определения и неформальное обсуждение подхода. Раздел 2 посвящен сравнению информативности априорных и апостериорных вероятностей. В разделе 3 изучаются алгебраические свойства апостериорной интеграции как бинарной операции на множестве вероятностей. В разделе 4 намечается исследование асимптотического поведения результатов апостериорной интеграции бесконечных последовательностей вероятностей.

\section{1. Основные определения и неформальное обсуждение.}

1.1. Базовые элементы. Далее $Z$ - непустое конечное множество, число элементов которого больше единицы, его элементы трактуются как допустимые элементарные события. Через П обозначено множество всех (элементарных) вероятностей на $Z$, понимаемых как неотрицательные функции на $Z$, суммы всех значений которых равны единице. Каждая вероятность $\pi \in \Pi$ определяет вероятностное пространство, понимаемое как пара $(Z, \pi)$. Через $\Pi^{+}$обозначаем множество вероятностей $\pi \in \Pi$, принимающих положительные значения. Через $\bar{\pi}$ обозначаем равномерную вероятность на $Z$, принимающую постоянное значение $1 /|Z|$ (здесь и далее $|E|$ - количество элементов конечного множества $E)$. Вероятность $\pi \in \Pi$ называем сосредоточенной в точке $z \in Z$, если $\pi(z)=1$. Вероятность $\pi \in \Pi$ называем сосредоточенной, если она сосредоточена в какой-либо точке. Для каждой вероятности 
$\pi \in \Pi$ полагаем $Z^{+}(\pi)=\{z \in Z: \pi(z)>0\}$. Множество П рассматриваем как метрическое пространство с естественной среднеквадратичной метрикой $\left(\pi_{1}, \pi_{2}\right) \mapsto\left[\sum_{z \in Z}\left|\pi_{1}(z)-\pi_{2}(z)\right|^{2}\right]^{1 / 2} ;$ ясно, что $\Pi-$ компакт. Для произвольного натурального $k$ рассматриваем $\Pi^{k}$ как произведение $k$ экземпляров метрического пространства П; всякое подмножество $\Pi^{k}$ трактуем как его метрическое подпространство. Непрерывность функций, определенных на подмножествах $\Pi^{k}$ и принимающих либо действительные значения, либо значения из П, понимаем в смысле вышеопределенных метрических пространств.

1.2. Неформальное обсуждение. Предположим, что некоторый детерминированный, априори зафиксированный, но неизвестный исследователю элемент $z^{0} \in Z$ наблюдается им с использованием $n$ методов наблюдения, которые мы пронумеруем $1, \ldots, n$. Каждый метод $i$ неточен в том смысле, что представляет $z^{0}$ в виде вероятностного распределения (вероятности) $\pi_{i}$ на $Z$. Для всякого $z \in Z$ значение $\pi_{i}(z)$ вероятности $\pi_{i}$ естественно трактовать как эмпирическую частоту регистрации элемента $z$ (в качестве $z^{0}$ ) в большой серии наблюдений, осуществляемых с помощью метода $i$. Вероятности $\pi_{i}, \ldots, \pi_{n}$ называем априорнылми вероятностями или априорныли вероятностными оценками наблюдаемого элемента $z^{0}$.

Будем рассматривать апостериорную ситуацию, сложившуюся после проведения наблюдений. Интересующая нас задача состоит в том, чтобы, отправляясь от априорных вероятностных оценок, синтезировать уточненную, интегральную информацию об элементе $z^{0}$.

Предполагаемый ниже подход к решению этой нестрого сформулированной задачи опирается на предположение о взаимной независимости методов наблюдения, точнее - на предположение о том, что распределение результатов $\left(z_{1}, \ldots, z_{n}\right) \in Z^{n}$ наблюдений, доставляемых методами $1, \ldots, n$, описывается пространством-произведением $\left(Z^{n}, P\right)=$ $\left(Z, \pi_{1}\right) \times \cdots \times\left(Z, \pi_{n}\right)$, где $P=\pi_{1} \times \cdots \times \pi_{n}$.

Данное предположение требует пояснения. Оно может выглядеть необоснованным, если наблюдаемый элемент имеет статистическую природу - является вариабельным элементарным событием в некотором нетривиальном вероятностном пространстве. Мы полагаем, что этот случай находится за рамками настоящей работы: как отмечено выше, наблюдаемый элемент $z^{0} \in Z$ предполагается априори зафиксированным - хотя и неизвестным исследователю. В этом случае предположение о независимости методов наблюдения - в обозначенном выше понимании - отражает достаточно типичное множество ситуаций (и подразумевается во многих прикладных работах, часть из них процитирована во введении - см. [5]-[8], [10]). Соответствующая модель наблюдения может быть описана следующим образом. Внутри устройства, используемого для многократного наблюдения одного и того же 
фиксированного элемента, помещается «игрок-помеха». При каждом акте использования устройства «игрок-помеха» производит случайное испытание, с тем чтобы «возмутить» наблюдение - сформировать переход от истинного наблюдаемого элемента к (вообще говоря) другому элементу - результату наблюдения; «игроки-помехи», находящиеся в разных устройствах, действуют независимо. Предположим, например, что наблюдаемый истинный элемент есть 2, «игрок-помеха», помещающийся в первом устройстве, формирует отклонения от истинного элемента размеров 0 и 1 с вероятностями $p_{1}(0)$ и $p_{1}(1)=1-p_{1}(0)$ соответственно, а «игрок-помеха», помещающийся во втором устройстве и никак не связанный с первым «игроком-помехой», формирует такие же отклонения от истинного элемента 2 с вероятностями $p_{2}(0)$ и $p_{2}(1)=1-p_{2}(0)$ соответственно. Тогда вероятностные пространства $\left(Z, \bar{p}_{1}\right)$ и $\left(Z, \bar{p}_{2}\right)$, где $Z=\{2,3\}, \bar{p}_{1}(z)=p_{1}(z-2), \bar{p}_{2}(z)=p_{2}(z-2)$ $(z \in Z)$, описывают распределения результатов наблюдения истинного элемента 2 с помощью первого и второго устройств соответственно, а их произведение $\left(Z^{2}, \bar{p}\right)=\left(Z, \bar{p}_{1}\right) \times\left(Z, \bar{p}_{2}\right)$, где $\bar{p}=\bar{p}_{1} \times \bar{p}_{2}$, описывает распределение пар $\left(z_{1}, z_{2}\right)$ результатов наблюдения, доставляемых двумя устройствами (здесь на первом месте стоит результат наблюдения с помощью первого устройства, на втором месте - результат наблюдения с помощью второго устройства). Исследователь, не зная, какой из двух элементов множества $Z$ является истинным, и не зная значений $\bar{p}_{1}(2)$ и $\bar{p}_{1}(3)$, аппроксимирует эти значения соответственно частотами $\pi_{1}(2)$ и $\pi_{1}(3)$ появления значений 2 и 3 в большой серии наблюдений с применением первого устройства; аналогичным образом он аппроксимирует неизвестные ему значения $\bar{p}_{2}(2)$ и $\bar{p}_{2}(2)$ частотами $\pi_{2}(2)$ и $\pi_{2}(3)$ появления значений 2 и 3 в большой серии наблюдений с применением второго устройства. Функции $\pi_{1}$ и $\pi_{2}$, понимаемые как вероятности на $Z$, принимаются исследователем за априорные вероятностные оценки неизвестного ему наблюдаемого истинного элемента, а пространствопроизведение $\left(Z^{2}, P\right)=\left(Z, \pi_{1}\right) \times\left(Z, \pi_{2}\right)$ - за (аппроксимационную) вероятностную модель, описывающую распределение пар $\left(z_{1}, z_{2}\right)$ результатов наблюдения, доставляемых двумя устройствами. Вероятность $P$ служит, понятно, весьма точной аппроксимацией указанной выше вероятности $\bar{p}$, характеризующей действительное распределение пар результатов наблюдения. На этом основании исследователь принимает пространство-произведение $\left(Z^{2}, P\right)$ за достаточно точное описание действительного распределения пар $\left(z_{1}, z_{2}\right)$ результатов наблюдений, доставляемых первым и вторым устройствами. В рассматриваемой здесь схеме (огрубленной или, наоборот, идеализированной - соответствующей бесконечным сериям наблюдений) исследователь отвлекается от аппроксимационного характера находящихся в его распоряжении вероятностей $\pi_{1}, \pi_{2}$ и $P=\pi_{1} \times \pi_{2}$ и использует их для решения вопроса об 
апостериорной интеграции априорных вероятностных оценок, доставляемых первым и вторым устройствами.

Предлагаемый подход к решению задачи об интеграции априорных вероятностных оценок исходит из того тривиального обстоятельства, что в апостериорной ситуации результаты $z_{1}, \ldots, z_{n} \in Z$ однократных наблюдений, доставляемые соответственно методами $1, \ldots, n$, являются верными тогда и только тогда, когда $z^{0}=z_{1}=\cdots=z_{n}$. Поскольку элемент $z^{0}$ неизвестен, выполнение равенств $z_{1}=\cdots=z_{n}$ есть необходимое условие апостериорной непротиворечивости результатов $z_{1}, \ldots, z_{n}$. Событие

$$
A=\left\{\left(z_{1}, \ldots, z_{n}\right) \in Z^{n}: z_{1}=\cdots=z_{n}\right\}=\{(z, \ldots, z): z \in Z\}
$$

в пространстве-произведении $\left(Z^{n}, P\right)=\left(Z, \pi_{1}\right) \times \cdots \times\left(Z, \pi_{n}\right)$, где $P=\pi_{1} \times$ $\times \pi_{n}$, выделяет все апостериорно непротиворечивые комбинации результатов наблюдений; все остальные комбинации $\left(z_{1}, \ldots, z_{n}\right)$ результатов наблюдений взаимно противоречивы и, следовательно, несут заведомо неверную информацию о наблюдаемом элементе. На этом основании заключаем, что в апостериорной ситуации в пространстве-произведении $\left(Z^{n}, P\right)$ с достоверностью реализуется событие $A$. Будем называть его апостериорньлм собьтием. Имеем

$$
P(A)=\sum_{z \in Z} \pi_{1}(z) \cdots \pi_{n}(z)
$$

Если $P(A)=0$, то методы $1, \ldots, n$ противоречивы в том смысле, что для каждого $z \in Z$ по крайней мере один из методов, $i$, допускает нулевую вероятность того, что $z=z^{0}: \pi_{i}(z)=0$.

Пусть методы $1, \ldots, n$ непротиворечивь, т.е. $P(A)>0$. На пространстве-произведении $\left(Z^{n}, P\right)$ рассмотрим условную вероятность $P(\cdot \mid$ $A)$ при условии апостериорного события $A$ :

$$
P((z, \ldots, z) \mid A)=\frac{\pi_{1}(z) \cdots \pi_{n}(z)}{P(A)} \quad(z \in Z) .
$$

Поскольку условная вероятность $P(\cdot \mid A)$ состредоточена на $A$ - «диагонали» в $Z^{n}$, все элементы которой имеют одинаковые компоненты, мы отождествим $P(\cdot \mid A)$ с вероятностью на $Z$; для последней примем обозначение $\pi_{1} \cdot \ldots \cdot \pi_{n}$; таким образом,

$$
\left(\pi_{1} \cdot \ldots \cdot \pi_{n}\right)(z)=P((z, \ldots, z) \mid A) \quad(z \in Z)
$$

Будем называть $\pi_{1} \cdot \ldots \cdot \pi_{n}$ результатом апостериорной интеграчии априорных вероятностей $\pi_{1}, \ldots, \pi_{n}$, а переход от $\pi_{1}, \ldots, \pi_{n} \mathrm{~K} \pi_{1} \cdot \ldots \cdot \pi_{n}$ anoстериорной интеграцией $\pi_{1}, \ldots, \pi_{n}$. 
Как видим, в вероятностном пространстве $\left(Z, \pi_{1} \cdot \ldots \cdot \pi_{n}\right)$ для каждого $z \in Z$ вероятность того, что $z$ есть истинный наблюдаемый элемент (вероятность того, что $\left.z^{0}=z\right)$, пропорциональна вероятности $\pi_{1}(z) \cdots \pi_{n}(z)$ того, что все методы наблюдения допускают, что $z$ есть истинный наблюдаемый элемент (что $z^{0}=z$ ), одновременно. Значение $\pi_{1}(z) \cdots \pi_{n}(z)$ есть «мера консенсуса» между методами $1, \ldots, n$ относительно того, что $z^{0}=z$. В формировании «меры консенсуса» $\pi_{1}(z) \cdots \pi_{n}(z)$ все методы равноправны, и каждый метод $i$ имеет «право вето» в том смысле, что при $\pi_{i}(z)=0$ «мера консенсуса» принимает нулевое значение. Мы принимаем, что апостериорная вероятность $\pi_{1} \cdot \ldots \cdot \pi_{n}$ доставляет искомую интегральную информацию о наблюдаемом элементе $z^{0}$, вытекающую из апостериорного анализа результатов его наблюдения с помощью методов $1, \ldots, n$.

Предложенный метод апостериорной интеграции априорных вероятностных оценок основан на самоочевидном логическом факте - факте заведомой реализации обозначенного выше апостериорного события $A$. Это отличает его от методов интеграции, традиционно применяемых в исследованиях социально-экономических систем и систем окружающей среды и часто сводящихся к правдоподобному обоснованию выбора коэффициентов выпуклых комбинаций априорных вероятностных оценок. В этой связи предложенный метод апостериорной интеграции может быть более эффективным, чем метод выпуклых комбинаций. Приведем поясняющий пример.

П р и м е р 1.1. Данный пример мотивирован исследованиями по классификации участков суши по типам (лес, травяное покрытие, пашня, пустыня и т.д.) посредством предъявления их спутниковых снимков, не доставляющих необходимой информации, многочисленным экспертам (см. http://www.geo-wiki.org/). Пусть $Z-$ конечное множество рассматриваемых типов суши и $z^{0} \in Z$ - тип конкретного участка. Для оценки (неизвестного нам) типа $z^{0}$ этого участка привлекаются $n$ независимых групп экспертов, каждый из которых, как предполагается, обладает дополнительными знаниями, позволяющими ему сделать обоснованное заключение относительно типа исследуемого участка. Распределение заключений экспертов, входящих группу с номером $i$ $(i=1, \ldots, n)$, представляет собой вероятность на $Z$; примем ее за априорную вероятностную оценку $\pi_{i}$; будем предполагать, что $\pi_{i}(z)>0$ для каждого $z \in Z$. Так как экспертные группы $1, \ldots, n$ независимы, принимаем, что распределение всех наборов $\left(z_{1}, \ldots, z_{n}\right)$ заключений, доставляемых этими группами, описывается пространством-произведением $\left(Z^{n}, P\right)=\left(Z, \pi_{1}\right) \times \cdots \times\left(Z, \pi_{n}\right)$. В качестве результата апостериорной обработки априорных вероятностных оценок $\pi_{1}, \ldots, \pi_{n}$ рассмотрим результат их интеграции, $\pi_{1} \cdot \ldots \cdot \pi_{n}$. Предположим, что среди экспертных групп $i$ есть «правильно распознающие» - такие, в которых процент 
экспертов, заключающих, что тип исследуемого участка есть $z^{0}$, максимален: $\pi_{i}\left(z^{0}\right)>\pi_{i}(z)$ для всех $z \in Z$, отличных от $z^{0}$. Множество всех таких групп обозначим через $G^{+}$, множество всех остальных групп через $G^{-}$. Допустим, что для каждой группы $i \in G^{+}$при всех $z \in Z$, отличных от $z^{0}$, выполняется неравенство $\pi_{i}\left(z^{0}\right)>q \pi_{i}(z)$, где $q>1$, а для каждой группы $j \in G^{-}$при всех $z \in Z$, отличных от $z^{0}$, выполняется неравенство $\pi_{j}\left(z^{0}\right)>r \pi_{j}(z)$, где $r \in(0,1)$. Пусть $m$ - число групп класса $G^{+}$. Положим $\pi^{+}(z)=\prod_{i \in G^{+}} \pi_{i}(z), \pi^{-}(z)=\prod_{i \in G^{-}} \pi_{i}(z)(z \in Z)$. Для каждого $z \in Z$ имеем

$$
\left(\pi_{1} \cdot \ldots \cdot \pi_{n}\right)(z)=\frac{\pi_{1}(z) \cdots \pi_{n}(z)}{\sum_{y \in Z} \pi_{1}(y) \cdots \pi_{n}(y)}=\left(1+\sum_{y \in Z \backslash\{z\}} \frac{\pi^{+}(y) \pi^{-}(y)}{\pi^{+}(z) \pi^{-}(z)}\right)^{-1}
$$

при этом

$$
\left(\pi_{1} \cdot \ldots \cdot \pi_{n}\right)\left(z^{0}\right)>\frac{1}{1+(n-1) / q^{m} r^{n-m}}>\frac{1}{1+\varepsilon}>1-\varepsilon
$$

для произвольно малого $\varepsilon>0$, если $q^{m} r^{n-m}>(n-1) / \varepsilon$; последнее неравенство выполняется, например, если $q r^{\alpha}>1$, где $\alpha>0$, количество $n$ экспертных групп достаточно велико и $n-m<\alpha m$; в самом деле, в этом случае

$$
\frac{q^{m} r^{n-m}}{n-1}=\frac{q^{m} r^{\alpha(n-m) / \alpha}}{n-1}>\frac{q^{m} r^{\alpha m}}{n-1}=\frac{\left(q r^{\alpha}\right)^{m}}{n-1}>\frac{\left(q r^{\alpha}\right)^{m}}{(\alpha+1) m-1},
$$

что стремится к бесконечности при стремлении $n$ (вместе с $m$ ) к бесконечности.

Теперь рассмотрим в качестве результата апостериорной обработки априорных вероятностных оценок $\pi_{1}, \ldots, \pi_{n}$ их произвольную выпуклую комбинацию $\pi=a_{1} \pi_{1}+\cdots+a_{n} \pi_{n}$; здесь $a_{1}, \ldots, a_{n} \geqslant 0, a_{1}+\cdots+a_{n}=1$. Для произвольного $i=1, \ldots, n$ имеем

$$
\pi_{i}\left(z_{0}\right) \leqslant 1-(N-1) \gamma_{i}
$$

где $N$ - число элементов множества $Z$ и $\gamma_{i}=\min _{z \in Z \backslash\left\{z^{0}\right\}} \pi_{i}(z)$; при этом, очевидно, $\gamma_{i}(N-1+r) \leqslant \sum_{z \in Z} \pi_{i}(z)=1$. Сообразуясь с последним ограничением, предположим, что для всех $i=1, \ldots, n$ выполняется неравенство $\gamma_{i}>\beta /(N-1+r)$, где $\beta \in(0,1)$. Тогда

$$
\pi\left(z_{0}\right) \leqslant \max _{i=1, \ldots, n} \pi_{i}\left(z_{0}\right)<1-\frac{N-1}{N-1+r} \beta .
$$

Правая часть этой верхней оценки, при выполнении всех сделанных выше предположений относительно $q, r$ и $\gamma_{i}(i=1, \ldots, n)$, меньше правой части полученной выше нижней оценки $1-\varepsilon$ для значения $\left(\pi_{1} \cdot \ldots \cdot \pi_{n}\right)\left(z^{0}\right)$, 
если $\varepsilon$ достаточно мало (последнее подразумевает, что $n$ достаточно велико).

Таким образом, в рассмотренном примере результат обработки априорных вероятностных оценок, выполненный в соответствии с предложенным методом, более предпочтителен, чем результат из обработки, полученный с помощью взятия из выпуклой комбинации.

1.3. Определения. Приведем строгие определения. Для произвольного натурального $n>1$ будем говорить, что вероятности $\pi_{1}, \ldots, \pi_{n} \in \Pi$ противоречивьи, если $\pi_{1}(z) \cdots \pi_{n}(z)=0$ для всех $z \in Z$; в противном случае будем говорить, что вероятности $\pi_{1}, \ldots, \pi_{n}$ непротиворечивы; через $\Pi^{(n)}$ будем обозначать множество всех $\left(\pi_{1}, \ldots, \pi_{n}\right) \in \Pi^{n}$ таких, что $\pi_{1}, \ldots, \pi_{n}$ непротиворечивы.

3 а м е ч а н и е 1.1. Очевидны утверждения: (i) $\left(\pi_{1}, \ldots, \pi_{n}\right) \in \Pi^{(n)}$ для всякой вероятности $\pi \in \Pi$ и всякого натурального $n>1$; (ii) $\left(\pi_{1}, \pi_{2}\right) \in \Pi^{(2)}$ для любых вероятностей $\pi_{1} \in \Pi$ и $\pi_{2} \in \Pi^{+}$; (iii) если $\left(\pi_{1}, \ldots, \pi_{n}\right) \in \Pi^{(n)}$, то $\left(\pi_{i_{1}}, \ldots, \pi_{i_{n}}\right) \in \Pi^{(n)}$ для всякой перестановки $\left(i_{1}, \ldots, i_{n}\right)$ в $(1, \ldots, n) ;(\mathrm{iv})\left(\Pi^{+}\right)^{n} \subset \Pi^{(n)}$ для всякого натурального $n>1$.

Следуя предварительному определению, данному в предыдущем пункте, для произвольного натурального $n \geqslant 2$ рассмотрим отображение $\left(\pi_{1}, \ldots, \pi_{n}\right) \mapsto \pi_{1} \cdot \ldots \cdot \pi_{n}: \Pi^{(n)} \mapsto \Pi$ такое, что

$$
\left(\pi_{1} \cdot \ldots \cdot \pi_{n}\right)(z)=\frac{\pi_{1}(z) \cdots \pi_{n}(z)}{\sum_{z^{\prime} \in Z} \pi_{1}\left(z^{\prime}\right) \cdots \pi_{n}\left(z^{\prime}\right)} \quad(z \in Z)
$$

для каждого набора $\left(\pi_{1}, \ldots, \pi_{n}\right) \in \Pi^{(n)}$. Данное отображение назовем $n$-кратной апостериорной интеграцией (кратко, интеграцией); для каждого набора $\left(\pi_{1}, \ldots, \pi_{n}\right) \in \Pi^{(n)}$ вероятность $\pi_{1} \cdot \ldots \cdot \pi_{n}$ будем называть результатом апостериорной интеграции (кратко, результатом интеграции) вероятностей $\pi_{1}, \ldots, \pi_{n}$.

3 а м е ч а н и е 1.2. Легко видеть, что $n$-кратная апостериорная интеграция непрерывна для любого натурального $n \geqslant 2$.

\section{2. Интеграция и оценки информативности.}

2.1. Интеграция и экстремальные элементы. Рассмотрим переход от априорной вероятностной оценки $\pi_{1} \in \Pi$ к апостериорной вероятностной оценки $\pi_{1} \cdot \pi_{2}$ получаемой в результате интеграции вероятности $\pi_{1}$ с какой-либо вероятностью $\pi_{2} \in$ П. Два простейших наблюдения отразим в следующем замечании.

3 а м е ч а н и е 2.1. (i) Интеграция произвольной вероятности $\pi \in \Pi$ с равномерной вероятностью $\bar{\pi}$ оставляет $\pi$ без изменения: $\pi \cdot \bar{\pi}=$ $\bar{\pi} \cdot \pi=\pi$. (ii) Если $\left(\pi, \pi_{*}\right) \in \Pi^{(2)}$ и вероятность $\pi_{*}$ сосредоточена, то интеграция $\pi$ с $\pi_{*}$ переводит $\pi$ в $\pi_{*}: \pi \cdot \pi_{*}=\pi_{*} \cdot \pi=\pi_{*}$.

Еще одно простое наблюдение состоит в том, что в типичных ситуациях после интеграции априорной вероятности $\pi_{1}$ с вероятностью 
$\pi_{2}$ вероятность элемента, наиболее вероятного в смысле вероятностного пространства $\left(Z, \pi_{2}\right)$, возрастает, а вероятность элемента наименее вероятного в смысле этого вероятностного пространства, убывает.

Лемма 2.1. Пусть $\left(\pi_{1}, \pi_{2}\right) \in \Pi^{(2)}, z^{*}-$ точка максимума вероятности $\pi_{2}, \pi_{1}\left(z^{*}\right)>0$ и существует элемент $z \in Z$, для которого $\pi_{2}(z)<\pi_{2}\left(z^{*}\right) u \pi_{1}(z) \pi_{2}(z)>0$. Тогда $\left(\pi_{1} \cdot \pi_{2}\right)\left(z^{*}\right)>\pi_{1}\left(z^{*}\right)$.

Д ок а з а т ель с т в о. Пусть $Z^{*}=\left\{z^{\prime} \in Z: \pi_{2}\left(z^{\prime}\right)=\pi_{2}\left(z^{*}\right)\right\}$. Заметим, что $z \in Z \backslash Z^{*}$. По определению

$$
\begin{aligned}
\left(\pi_{1} \cdot \pi_{2}\right)\left(z^{*}\right) & =\frac{\pi_{1}\left(z^{*}\right) \pi_{2}\left(z^{*}\right)}{\sum_{z^{\prime} \in Z} \pi_{1}\left(z^{\prime}\right) \pi_{2}\left(z^{\prime}\right)} \\
& =\frac{\pi_{1}\left(z^{*}\right)}{\sum_{z^{\prime} \in Z^{*}} \pi_{1}\left(z^{\prime}\right)+\pi_{1}(z) q(z)+\sum_{z^{\prime} \in Z \backslash\left(Z^{*} \cup\{z\}\right)} \pi_{1}\left(z^{\prime}\right) q\left(z^{\prime}\right)},
\end{aligned}
$$

где

$$
q\left(z^{\prime}\right)=\frac{\pi_{2}\left(z^{\prime}\right)}{\pi_{2}\left(z^{*}\right)} \quad\left(z^{\prime} \in Z\right) .
$$

Так как $\pi_{2}\left(z^{*}\right)=\max _{z \in Z}$, имеем $q\left(z^{\prime}\right)<1$ для всех $z^{\prime} \in Z \backslash Z^{*}$, что, вместе с неравенством $\pi_{1}(z) \pi_{2}(z)>0$, влечет оценку $\pi_{1}(z) q(z)<\pi_{1}(z)$. Следовательно, знаменатель в правой части (2.1) меньше, чем $\sum_{z^{\prime} \in Z} \pi_{1}\left(z^{\prime}\right)=1$. Теперь (2.1) и предположение $\pi_{1}\left(z^{*}\right)>0$ дают $\left(\pi_{1} \cdot \pi_{2}\right)\left(z^{*}\right)<\pi_{1}\left(z^{*}\right)$. Лемма доказана.

Аналогичное рассуждение ведет к следующему симметричному утверждению.

Лемма 2.2. Пусть $\left(\pi_{1}, \pi_{2}\right) \in \Pi^{(2)}, z_{*}-$ точка минимума вероятности $\pi_{2}, \pi_{1}\left(z_{*}\right)>0$ и существует элемент $z \in Z$, для которого $\pi_{2}(z)>\pi_{2}\left(z_{*}\right) u \pi_{1}(z) \pi_{2}(z)>0$. Тогда $\left(\pi_{1} \cdot \pi_{2}\right)\left(z_{*}\right)<\pi_{1}\left(z_{*}\right)$.

2.2. Меры сосредоточенности. Для вероятностей из класса П рассмотрим числовые индикаторы, достигающие наибольших значений на сосредоточенных вероятностях; будем называть их мерами сосредоточенности. Меры сосредоточенности можно интерпретировать как показатели информативности вероятностей. Если результат интеграции двух априорных вероятностей (априорных оценок, доставляемых независимыми методами) имеет более высокую меру сосредоточенности, чем каждая их них, мы имеем основание утверждать, что априорные вероятностные оценки согласованы: взаимодействуя, они несут больше информации, чем каждая из них в отдельности. В противоположной ситуации, когда результат интеграции двух априорных вероятностей имеет меньшую меру сосредоточенности, чем каждая из них, априорные оценки находятся в конфликте друг с другом, одну их них, скорее всего, следует отбросить. Наконец, в промежуточной ситуации, когда мера сосредоточенности вероятности, получающейся в результате интеграции 
двух априорных вероятностей, выше минимума и ниже максимума их мер сосредоточенности, можно говорить об асимметричной взаимосвязи априорных вероятностных моделей: одна из них уточняет другую, но не наоборот. С практической точки зрения наибольший интерес представляет первая из обозначенных выше ситуаций, в которой результат интеграции априорных вероятностей $\pi_{1}$ и $\pi_{2}$ имеет большую меру сосредоточенности, чем каждая из них; в этой ситуации мы будем говорить, что пара $\left(\pi_{1}, \pi_{2}\right)$ вероятностей совместна (относительно заданной меры сосредоточенности).

Итак, под мерой сосредоточенности будем понимать произвольную непрерывную функцию $\mu: \Pi \mapsto(-\infty, 1]$ такую, что $\mu(\pi)=1$ тогда и только тогда, когда вероятность $\pi$ сосредоточена. Про пару $\left(\pi_{1}, \pi_{2}\right) \in \Pi^{2}$ будем говорить, что она совместна относительно меры сосредоточенности $\mu$, если $\left(\pi_{1}, \pi_{2}\right) \in \Pi^{(2)}$ и $\mu\left(\pi_{1} \cdot \pi_{2}\right)>\max \left\{\mu\left(\pi_{1}\right), \mu\left(\pi_{2}\right)\right\}$, и несовместна относительно $\mu$, если $\left(\pi_{1}, \pi_{2}\right) \in \Pi^{(2)}$ и $\mu\left(\pi_{1} \cdot \pi_{2}\right)<\min \left\{\mu\left(\pi_{1}\right), \mu\left(\pi_{2}\right)\right\}$.

Простейшей мерой сосредоточенности является функция $\pi \mapsto$ $\max _{z \in Z} \pi(z)$, будем называть ее max-мерой сосредоточенности. В данном, начальном, исследовании будем рассматривать только maх-меру сосредоточенности.

3 а м е ч а н и е 2.2. Приведем несколько примеров других мер сосредоточенности: (i) $\pi \mapsto \max _{z \in Z} \pi(z)-\min _{z \in Z} \pi(z)$; (ii) $\pi \mapsto \sum_{z \in Z} \pi^{k}(z)$, где $k>1$; (iii) $\pi \mapsto 1-\sum_{z \in Z}\left[\xi(z)-\sum_{z^{\prime} \in Z} \xi\left(z^{\prime}\right) \pi\left(z^{\prime}\right)\right]^{2} \pi(z)$, где $\xi-$ произвольная действительная взаимно однозначная функция на $Z$; значение данной меры сосредоточенности на вероятности $\pi \in \Pi$ есть дисперсия случайной величины $\xi$ на $(Z, \pi)$; (iv) $\pi \mapsto 1+\sum_{z \in Z} \pi(z) \log \pi(z)$ (при $\pi(z)=0$ мы полагаем $\pi(z) \log \pi(z)=0)$; последняя сумма, взятая с противоположным знаком известна как энтропия вероятности $\pi$.

3 а м е ч а н и е 2.3. Ясно, что минимальное значение тах-меры сосредоточенности есть $1 /|Z|$; оно достигается на равномерной вероятности $\bar{\pi}$ и только на ней.

Для краткости пару $\left(\pi_{1}, \pi_{2}\right) \in \Pi^{2}$, совместную (соответственно несовместную) относительно тах-меры сосредоточенности, будем называть тах-совместной (соответственно тах-несовместной).

Используя лемму 2.1, отметим типичную ситуацию, когда пара $\left(\pi_{1}, \pi_{2}\right) \in \Pi^{(2)}$ является max-совместной. Эта ситуация предполагает, что множества элементарных событий, наиболее вероятных в вероятностных пространствах $\left(Z, \pi_{1}\right)$ и $\left(Z, \pi_{2}\right)$, имеют непустое пересечение.

Теорема 2.1. Пусть $\left(\pi_{1}, \pi_{2}\right) \in \Pi^{(2)}$ и существует элемент $z^{*} \in Z$, максимизирующий на $Z$ каждую из вероятностей $\pi_{1}$ и $\pi_{2}$. Справедливь следуюшие утверждения.

1. Элемент $z^{*}$ максимизирует $\pi_{1} \cdot \pi_{2}$ на $Z$.

2. Если существует элемент $z \in Z$ такой, что $0<\pi_{1}(z)<$ 
$\max _{z^{\prime} \in Z} \pi_{1}\left(z^{\prime}\right)$ u $0<\pi_{2}(z)<\max _{z^{\prime} \in Z} \pi_{2}\left(z^{\prime}\right)$, mo napa $\left(\pi_{1}, \pi_{2}\right)$ maxсовместна.

Д о к а з а т е л ь с т в о. Утверждение 1 следует непосредственно из определения результата интеграции $\pi_{1} \cdot \pi_{2}$. Докажем утверждение 2. Очевидно, что $\pi_{1}\left(z^{*}\right)>0$. По предположению $\pi_{2}(z)<\pi_{2}\left(z^{*}\right)$ и $\pi_{1}(z) \pi_{2}(z)>0$. Таким образом, выполнены все предположения леммы 2.1. Применяя эту лемму, получаем, что $\left(\pi_{1} \cdot \pi_{2}\right)\left(z^{*}\right)>\pi_{1}\left(z^{*}\right)=$ $\max _{z^{\prime} \in Z} \pi_{1}\left(z^{\prime}\right)$. Меняя в этом рассуждении местами $\pi_{1}$ и $\pi_{2}$, приходим к симметричным соотношениям $\left(\pi_{1} \cdot \pi_{2}\right)\left(z^{*}\right)>\pi_{2}\left(z^{*}\right)=\max _{z^{\prime} \in Z} \pi_{2}\left(z^{\prime}\right)$. Доказательство закончено.

Из теоремы 2.1 сразу получаем следующее утверждение.

Следствие 2.1. Для каждой несосредоточенной неравномерной вероятности $\pi \in \Pi$ пара $(\pi, \pi)$ является $\operatorname{max-совместной.~}$

Распространим понятие тах-совместности с пар $\left(\pi_{1}, \pi_{2}\right) \in \Pi^{(2)}$ на $n$-местные наборы $\left(\pi_{1}, \ldots, \pi_{n}\right) \in \Pi^{(n)}$. Для каждого натурального $n \geqslant 2$ будем говорить, что набор $\left(\pi_{1}, \ldots, \pi_{n}\right) \in \Pi^{(n)}$ max-совместен, если

$$
\max _{z \in Z}\left(\pi_{1} \cdot \ldots \cdot \pi_{n}\right)(z)>\max \left\{\max _{z \in Z} \pi_{1}(z), \ldots, \max _{z \in Z} \pi_{n}(z)\right\}
$$

Опираясь на теорему 2.1, нетрудно установить следующее.

Следствие 2.2. Пусть число $n \geqslant 2$ - натуральное, $\left(\pi_{1}, \ldots, \pi_{2}\right) \in$ $\Pi^{(n)}$ и существует элемент $z^{*} \in Z$, максимизирующий на $Z$ каждую из вероятностей $\pi_{1}, \ldots, \pi_{n}$. Справедливь следуюшие утверждения.

1. Элемент $z^{*}$ максимизирует $\pi_{1} \cdot \ldots \cdot \pi_{n}$ на $Z$.

2. Если существует элемент $z \in Z$ такой, что $0<\pi_{i}(z)<$ $\max _{z^{\prime} \in Z} \pi_{i}\left(z^{\prime}\right)$ для всех $i \in\{1, \ldots, n\}$, то набор $\left(\pi_{1}, \ldots, \pi_{n}\right)$ maxсовместен.

Следующее наблюдение, вытекающее из следствия 2.1, касается топологической структуры множеств вероятностей, инвариантных относительно интеграции. Будем говорить, что множество $G \subset$ П инвариантно относительно интеграчии, если для всяких $\pi_{1}, \pi_{2} \in G$ вероятности $\pi_{1}$ и $\pi_{2}$ непротиворечивы и $\pi_{1} \cdot \pi_{2} \in G$.

Теорема 2.2. Пусть непустое множество $G \subset$ П инвариантно относительно интеграчии и не содержит сосредоточенньх вероятностей. Тогда справедливо одно и только одно из следующих утверждений: (i) множество $G$ одноэлементно и его единственный элемент есть равномерная вероятность $\bar{\pi}$; (ii) $G$ не замкнуто в П.

Пусть множество $G$ одноэлементно и $\pi$ - его элемент. Тогда $\pi \cdot \pi=\pi$. Допустим, что $\pi \neq \bar{\pi}$. По следствию 2.1 пара $(\pi, \pi)$ maxсовместна, т.е. $\max _{z \in Z}(\pi \cdot \pi)(z)>\max _{z \in Z} \pi(z)$. Последнее невозможно, так как $\pi \cdot \pi=\pi$. Следовательно, $\pi=\bar{\pi}$. 
Пусть $G$ не одноэлементно. Тогда $G$ содержит неравномерную вероятность. Допустим, что $G$ замкнуто. Пусть $p=\sup _{\pi \in G} \max _{z \in Z} \pi(z)$. Поскольку $G$ содержит неравномерную вероятность, $p$ больше, чем $1 /|Z|-$ значение, принимаемое равномерной вероятностью. Поскольку $G$ замкнуто, существует вероятность $\pi_{*} \in G$ такая, что $\max _{z \in Z} \pi_{*}(z)=p$. Из того, что $p>1 /|Z|$, следует, что $\pi_{*}$ не является равномерной. Так как по предположению $G$ не содержит сосредоточенных вероятностей, $\pi_{*}$ не является сосредоточенной. Тогда по следствию 2.1 пара $\left(\pi_{*}, \pi_{*}\right)$ max-совместна, т.е. $\max _{z \in Z}\left(\pi_{*} \cdot \pi_{*}\right)(z)>\max _{z \in Z} \pi_{*}(z)=p$. Но $\pi_{*} \cdot \pi_{*} \in G$, поэтому $\max _{z \in Z}\left(\pi_{*} \cdot \pi_{*}\right)(z) \leqslant p$. Полученное противоречие завершает доказательство.

Отметим следующее непосредственное следствие из теоремы 2.2.

Следствие 2.3. Пусть непустое множество $G \subset$ П инвариантно относительно интеграиии, не содержит сосредоточенных вероятностей и не является одноэлементным. Тогда $G$ не конечно.

В заключение данного пункта заметим, что ситуация, когда пара $\left(\pi_{1}, \pi_{2}\right) \in \Pi^{(2)}$ max-несовместна, подразумевает, что элементы, имеющие высокие вероятности в вероятностном пространстве $\left(Z, \pi_{1}\right)$, имеют малые вероятности в вероятностном пространстве $\left(Z, \pi_{2}\right)$ и наоборот, что говорит о качественном несоответствии вероятностных моделей $\pi_{1}$ и $\pi_{2}$. Ниже приведен простейший пример max-несовместной пары вероятностей.

П р и м е р 2.1. Пусть $Z=\left\{z_{1}, z_{2}\right\}, \pi_{1}\left(z_{1}\right)=3 / 4, \pi_{1}\left(z_{2}\right)=1 / 4$, $\pi_{2}\left(z_{1}\right)=1 / 4, \pi_{2}\left(z_{2}\right)=3 / 4$. Тогда $\left(\pi_{1} \cdot \pi_{2}\right)\left(z_{1}\right)=\left(\pi_{1} \cdot \pi_{2}\right)\left(z_{2}\right)=1 / 2<$ $3 / 4=\max _{z \in Z} \pi_{1}(z)=\max _{z \in Z} \pi_{2}(z)$.

2.3. Маргинальная мера. Для всякой несосредоточенной вероятности $\pi \in \Pi$ определим маргинальную меру вероятности $\pi \in \Pi$ как $\min _{z \in Z^{+}(\pi)} \pi(z)$.

Ясно, что для всякой не сосредоточенной вероятности $\pi \in \Pi$ ее маргинальная мера не превосходит $1 /|Z|$ и равна $1 /|Z|$ тогда и только тогда, когда вероятность $\pi$ равномерна $(\pi=\bar{\pi})$. В этом контексте, чем меньшую маргинальную меру имеет не сосредоточенная вероятность $\pi$, тем она «менее равномерна». Поскольку равномерная вероятность является наименее информативной среди всех вероятностей из П, вероятности с малыми маргинальными мерами можно интерпретировать как в некотором смысле более информативные, нежели вероятности с большими (близкими к $1 /|Z|$ ) маргинальными мерами.

Будем говорить, что пара $\left(\pi_{1}, \pi_{2}\right) \in \Pi^{(2)}$ не сосредоточенных вероятностей маргинально совместна, если

$$
\min _{z^{\prime} \in Z^{+}\left(\pi_{1}, \pi_{2}\right)}\left(\pi_{1} \cdot \pi_{2}\right)\left(z^{\prime}\right)<\min \left\{\min _{z^{\prime} \in Z^{+}\left(\pi_{1}\right)} \pi_{1}\left(z^{\prime}\right), \min _{z^{\prime} \in Z^{+}\left(\pi_{2}\right)} \pi_{2}\left(z^{\prime}\right)\right\}
$$


и маргинально несовместна, если выполняется противоположное строгое неравенство.

Лемма 2.2 позволяет описать типичную ситуацию, когда пара $\left(\pi_{1}, \pi_{2}\right) \in \Pi^{(2)}$ является маргинально совместной: данное свойство имеет место, если множества элементов, наименее вероятных в вероятностных пространствах $\left(Z, \pi_{1}\right)$ и $\left(Z, \pi_{2}\right)$, имеют непустое пересечение.

Теорема 2.3. Пусть вероятности $\pi_{1}, \pi_{2} \in \Pi$ не сосредоточень, $\left(\pi_{1}, \pi_{2}\right) \in \Pi^{(2)}$ и существует элемент $z_{*} \in Z$, минимизирующий на $Z^{+}\left(\pi_{1}\right)$ вероятность $p_{1}$ и на $Z^{+}\left(\pi_{2}\right)$ вероятность $p_{2}$. Тогда справедливь следующие утверждения.

1. Элемент $z_{*}$ минимизирует $\pi_{1} \cdot \pi_{2}$ на $Z^{+}\left(\pi_{1} \cdot \pi_{2}\right)$.

2. Если существует элемент $z \in Z$ такой, что

$$
\pi_{1}(z)>\min _{z^{\prime} \in Z^{+}\left(\pi_{1}\right)} \pi_{1}\left(z^{\prime}\right) \quad u \quad \pi_{2}(z)>\min _{z^{\prime} \in Z^{+}\left(\pi_{2}\right)} \pi_{2}\left(z^{\prime}\right),
$$

то пара $\left(\pi_{1}, \pi_{2}\right)$ маргинально совместна.

Доказательство данной теоремы аналогично доказательству теоремы 2.1. Из теоремы 2.3 сразу вытекает следующее.

Следствие 2.4. Для всякой не сосредоточенной не равномерной вероятности $\pi \in \Pi$ пара $(\pi, \pi)$ маргинально совместна.

Распространяя определение маргинальной совместности, будем говорить, что $n$-местный набор $\left(\pi_{1}, \ldots, \pi_{n}\right) \in \Pi^{(n)}$ не сосредоточенных вероятностей (здесь $n$ - натуральное число, большее единицы) маргинально совместен, если

$$
\min _{z \in Z}\left(\pi_{1} \cdot \ldots \cdot \pi_{n}\right)(z)<\min \left\{\min _{z \in Z} \pi_{1}(z), \ldots, \min _{z \in Z} \pi_{n}(z)\right\} .
$$

Приведем без доказательства естественное расширение теоремы 2.3.

Следствие 2.5. Пусть $n \geqslant 2$ - натуральное, вероятности $\pi_{1}, \ldots, \pi_{n} \in \Pi$ не сосредоточеньи, $\left(\pi_{1}, \ldots, \pi_{n}\right) \in \Pi^{(n)}$ и существует элемент $z_{*} \in Z$ такой, что для каждого $i=1, \ldots, n$ он минимизирует вероятность $\pi_{i}$ на множестве $Z^{+}\left(\pi_{i}\right)$. Тогда справедливь следующие утверждения.

1. Элемент $z_{*}$ минимизирует $\pi_{1} \cdot \ldots \cdot \pi_{n}$ на $Z^{+}\left(\pi_{1} \cdot \ldots \cdot \pi_{n}\right)$.

2. Если существует элемент $z \in Z$ такой, что

$$
\pi_{i}(z)>\max _{z^{\prime} \in Z} \pi_{i}\left(z^{\prime}\right) \quad \text { для всех } i \in\{1, \ldots, n\},
$$

то набор $\left(\pi_{1}, \ldots, \pi_{n}\right)$ маргинально совместен.

Ситуация, когда пара $\left(\pi_{1}, \pi_{2}\right) \in \Pi^{(2)}$ не сосредоточенных вероятностей маргинально несовместна, в определенном смысле аналогична ситуации, когда эта пара не является max-совместной: обе ситуации подразумевают, что элементарные события, имеющие высокие вероятности 
в вероятностном пространстве $\left(Z, \pi_{1}\right)$, имеют малые вероятности в вероятностном пространстве $\left(Z, \pi_{2}\right)$, и наоборот. Простейшую иллюстрацию доставляет пример 2.1 .

2.4. Мах-концентраторы. Вероятность $\pi \in \Pi$ назовем maxконцентратором для набора $\left(\pi_{1}, \ldots, \pi_{n}\right) \in \Pi^{n}$ (здесь $n-$ натуральное число, большее единицы), если пара $\left(\pi, \pi_{i}\right)$ max-совместна для каждого $i \in\{1, \ldots, n\}$. Таким образом, вероятностная оценка $\pi$, являющаяся max-концентратором для набора $\left(\pi_{1}, \ldots, \pi_{n}\right)$ оценок, повышает, посредством интеграции, max-меру сосредоточенности каждой из них.

Легко выделяются max-концентраторы для наборов попарно maxсовместных вероятностей.

Теорема 2.4. Пусть $n \geqslant 2$ - натуральное и набор $\left(\pi_{1}, \ldots, \pi_{n}\right) \in$ $\Pi^{n}$ вероятностей таков, что для любых различных $i, j \in\{1, \ldots, n\}$ пара $\left(\pi_{i}, \pi_{j}\right)$ является тах-совместной. Тогда для каждого $i \in\{1, \ldots, n\}$ вероятность $\pi_{i}$ есть тах-концентратор для набора $\left(\pi_{1}, \ldots, \pi_{n}\right)$.

Д о к а з а т е ль с т в о. Пусть $i, j \in\{1, \ldots, n\}$ и $j \neq i$. Имеем $\left(\pi_{i}, \pi_{i}\right) \in \Pi^{(2)}$ (см. замечание $\left.1.1,(\mathrm{i})\right)$, кроме того, $\left(\pi_{i}, \pi_{j}\right) \in \Pi^{(2)}$ по предположению. По предположению же пара $\left(\pi_{i}, \pi_{j}\right)$ max-совместна. Последняя исключает то, что вероятность $\pi_{i}$ равномерна (см. замечание $2.1,(\mathrm{i}))$. Значит, по следствию 2.1 пара $\left(\pi_{i}, \pi_{i}\right)$ maх-совместна. Доказательство закончено.

Следующая теорема показывает, что в типичных случаях вероятность, отдающая достаточно сильное предпочтение элементарному событию, имеющему ненулевую вероятность в каждом из вероятностных пространств $\left(Z, \pi_{1}\right), \ldots,\left(Z, \pi_{n}\right)$, является maх-концентратором для набора $\left(\pi_{1}, \ldots, \pi_{n}\right)$.

Теорема 2.5. Пусть $n \geqslant 2$ - натуральное, $\left(\pi_{1}, \ldots, \pi_{n}\right) \in \Pi^{n}$, вероятности $\pi_{1}, \ldots, \pi_{n}$ не сосредоточень и элемент $z_{*} \in Z$ таков, что $\pi_{i}\left(z_{*}\right)>0$ для всех $i \in\{1, \ldots, n\}$. Тогда всякая вероятность $\pi \in \Pi$ та$\kappa a я$, что $\pi\left(z_{*}\right)$ достаточно близко $к$ единиче, есть тах-концентратор для набора $\left(\pi_{1}, \ldots, \pi_{n}\right)$.

Д о к а з а т е ль с т в о. Возьмем вероятность $\pi_{*} \in \Pi$, сосредоточенную в $z_{*}$. Ясно, что $\left(\pi_{*}, \pi_{i}\right) \in \Pi^{(2)}$ для каждого $i \in\{1, \ldots, n\}$. Согласно замечанию 2.1 , (ii), для каждого $i \in\{1, \ldots, n\}$ имеем $\pi_{*} \cdot \pi_{i}=\pi_{*}$ и, следовательно, $\max _{z \in Z}\left(\pi_{*} \cdot \pi_{i}\right)(z)=1>\max _{z \in Z} \pi_{i}(z)$; последнее неравенство обусловлено тем, что вероятность $\pi_{i}$ не сосредоточена. Благодаря непрерывности интеграции (см. замечание 1.2) и непрерывности maxмеры сосредоточенности последнее неравенство сохраняется для всех $i \in\{1, \ldots, n\}$ при замене в нем вероятности $\pi_{*}$ на произвольную вероятность $\pi \in \Pi$ такую, что $\pi\left(z_{*}\right)$ достаточно близко к единице. Доказательство закончено. 
Следующая теорема касается наборов вероятностей, достаточно близких к равномерной вероятности.

Теорема 2.6. Пусть $n \geqslant 2$ - натуральное и вероятность $\pi \in \Pi$ не равномерна. Тогда $\pi$ есть тах-концентратор для любого набора $\left(\pi_{1}, \ldots, \pi_{n}\right) \in \Pi^{n}$ такого, что вероятности $\pi_{1}, \ldots, \pi_{n}$ достаточно близки к равномерной вероятности $\bar{\pi}$.

Д о к а з а т е л ь с т в о. Согласно замечанию 2.1, (i), имеет место равенство $\pi \cdot \bar{\pi}=\pi$. Тогда, с учетом того, что вероятность $\pi$ не равномерна, имеем $\max _{z \in Z}(\pi \cdot \bar{\pi})=\max _{z \in Z} \bar{\pi}(z)=1 /|Z|$. Вследствие непрерывности интеграции (см. замечание 1.1) и непрерывности тах-меры сосредоточенности последнее неравенство сохраняется после замены в нем равномерной вероятности $\bar{\pi}$ на произвольные вероятности $\pi_{1}, \ldots, \pi_{n} \in \Pi$, достаточно близкие к $\bar{\pi}$. Доказательство закончено.

Покажем, что если $n<|Z|$, то в типичных случаях для заданного набора $\left(\pi_{1}, \ldots, \pi_{n}\right)$ вероятностей можно найти тах-концентратор, достаточно близкий к равномерной вероятности. Интерпретируя это свойство содержательно, можно сказать, что оценку, доставляемую каждым из $n$ заданных методов наблюдения, возможно улучшить путем ее интеграции с оценкой, доставляемой одним и тем же дополнительным методом, информационное качество которого весьма низко.

Теорема 2.7. Пусть $N=|Z|$ и натуральное $n$ таково, что $2 \leqslant n \leqslant N$. Пусть $\pi_{1}, \ldots, \pi_{n} \in \Pi^{n}, Z=\left\{z_{1}, \ldots, z_{N}\right\}$, для каждого $i \in\{1, \ldots, n\} z_{k_{i}}-$ точка максимума вероятности $\pi_{i}$,

$$
v_{i k}=\left\{\begin{array}{ll}
0, & \text { если } k \neq k_{i}, \\
1, & \text { если } k=k_{i}
\end{array} \quad(i \in\{1, \ldots, n\}, k \in\{1, \ldots, N\})\right.
$$

и ранг матрищь

$$
A=\left(\begin{array}{cccc}
\pi_{1}\left(z_{1}\right)-v_{11} & \pi_{1}\left(z_{2}\right)-v_{12} & \ldots & \pi_{1}\left(z_{N}\right)-v_{1 N} \\
\ldots & \ldots & \ldots & \ldots \\
\pi_{n}\left(z_{1}\right)-v_{n 1} & \pi_{n}\left(z_{2}\right)-v_{n 2} & \ldots & \pi_{n}\left(z_{N}\right)-v_{n N} \\
1 & 1 & \ldots & 1
\end{array}\right)
$$

не меньше $n+1$. Тогда, каково бь ни било $\varepsilon>0$, для набора $\left(\pi_{1}, \ldots, \pi_{n}\right)$ существует тах-концентратор $\pi$ такой, что расстояние в П от $\pi$ до равномерной вероятности $\bar{\pi}$ меньше $\varepsilon$.

Д о к а з а т е ль с т в о. Для каждой вероятности $\pi \in$ П будем обозначать через $\pi^{*}$ вектор $\left(\pi\left(z_{1}\right), \ldots, \pi\left(z_{N}\right)\right) \in \mathbf{R}^{N}$ ее значений. Для каждого вектора $h=\left(h_{1}, \ldots, h_{N}\right) \in \mathbf{R}^{N}$ такого, что

$$
h_{1}+\cdots+h_{N}=0
$$

и каждого достаточно малого $\lambda>0$, очевидно, имеем

$$
\bar{\pi}^{*}+\lambda h \in \Pi^{*}=\left\{\pi^{*}: \pi \in \Pi\right\} .
$$


Для всякого вектора $p=\left(p+1, \ldots, p_{N}\right) \in \mathbf{R}^{N}$ с положительными компонентами положим

$$
g_{i k}(p)=\frac{\pi_{i}\left(z_{k}\right) p_{k}}{\sum_{j=1}^{N} \pi_{i}\left(z_{j}\right) p_{j}} \quad(i \in\{1, \ldots, n\}, k \in\{1, \ldots, N\}) .
$$

Ясно, что для любой вероятности $\pi \in \Pi$ с положительными значениями имеем

$$
g_{i k}\left(\pi^{*}\right)=\left(\pi_{i} \cdot \pi\right)\left(z_{k}\right) \quad(i \in\{1, \ldots, n\}, k \in\{1, \ldots, N\}),
$$

в частности,

$$
g_{i k}\left(\bar{\pi}^{*}\right)=\left(\bar{\pi}_{i} \cdot \pi\right)\left(z_{k}\right)=\pi\left(z_{k}\right) \quad(i \in\{1, \ldots, n\}, k \in\{1, \ldots, N\}) .
$$

Теперь, вспоминая, что (2.6) и (2.4) выполняются для всех $h \in \mathbf{R}^{N}$, удовлетворяющих (2.3), и всех достаточно малых $\lambda>0$, видим, что для завершения доказательства осталось показать, что существует вектор $h \in \mathbf{R}^{N}$ такой, что для всех достаточно малых $\lambda>0$ справедливы неравенства

$$
\max _{k=1, \ldots, N} g_{i k}\left(\bar{\pi}^{*}+\lambda h\right)>\max _{k=1, \ldots, N} \pi_{i}\left(z_{k}\right) \quad(i \in\{1, \ldots, n\}) .
$$

Для этого, в свою очередь, с учетом того, что $z_{k_{i}}$ при каждом $i \in$ $\{1, \ldots, n\}$ есть точка максимума вероятности $\pi_{i}$, достаточно установить, что при некотором $h \in \mathbf{R}^{N}$ и всех достаточно малых $\lambda>0$ верны неравенства

$$
g_{i k_{i}}\left(\bar{\pi}^{*}+\lambda h\right)>\pi_{i}\left(z_{k_{i}}\right) \quad(i \in\{1, \ldots, n\}) .
$$

Установим это. Заметим, что при заданном $h \in \mathbf{R}^{N}$ и всех достаточно малых $\lambda>0$ неравенства (2.8) равносильны неравенствам

$$
g_{i k_{i}}\left(\bar{\pi}^{*}\right)+\left\langle\operatorname{grad} g_{i k_{i}}\left(\bar{\pi}^{*}\right), h\right\rangle \lambda>\pi_{i}(z) i k_{i} \quad(i \in\{1, \ldots, n\})
$$

или, ввиду $(2.7)$ (где $\pi=\pi_{i}$ при $\left.i \in\{1, \ldots, n\}\right)$, неравенствам

$$
\left\langle\operatorname{grad} g_{i k_{i}}\left(\bar{\pi}^{*}\right), h\right\rangle>0 \quad(i \in\{1, \ldots, n\})
$$

здесь $\operatorname{grad} g_{i k}\left(\bar{\pi}^{*}\right)$ — градиент функции $p \mapsto g_{i k}(p)$ в точке $\bar{\pi}^{*}$ и $\langle\cdot, \cdot\rangle$ — скалярное произведение в $\mathbf{R}^{N}$.

Итак, покажем, что существует вектор $h \in \mathbf{R}^{N}$, удовлетворяющий равенству (2.3) и неравенствам (2.9). Обращаясь к (2.5), находим:

$$
\operatorname{grad} g_{i k_{i}}(\bar{p})=\left(\gamma_{i}^{(1)}, \ldots, \gamma_{i}^{(N)}\right)
$$


где

$$
\begin{aligned}
\gamma_{i}^{(k)} & =-\frac{\pi_{i}\left(z_{k_{i}}\right) \pi_{i}\left(z_{k}\right)(1 / N)}{\left(\sum_{j=1}^{N} \pi_{i}\left(z_{j}\right)(1 / N)\right)^{2}}=-N \pi_{i}\left(z_{k_{i}}\right) \pi_{i}\left(z_{k}\right) \quad \text { при } k \neq k_{i}, \\
\gamma_{i}^{\left(k_{i}\right)} & =\frac{\pi_{i}\left(z_{k_{i}}\right) \sum_{j=1}^{N} \pi_{i}\left(z_{j}\right)(1 / N)-\pi_{i}(z) \pi_{i}\left(z_{k}\right)(1 / N)}{\left(\sum_{j=1}^{N} \pi_{i}\left(z_{j}\right)(1 / N)\right)^{2}}=N\left[\pi_{i}\left(z_{k_{i}}\right)-\pi_{i}^{2}\left(z_{k_{i}}\right)\right]
\end{aligned}
$$

здесь учтено, что $\sum_{j=1}^{N} \pi_{i}\left(z_{j}\right)=1$. Теперь для произвольного $h=$ $\left(h_{1}, \ldots, h_{N}\right) \in \mathbf{R}^{N}$ имеем:

$$
\begin{aligned}
& \left\langle\operatorname{grad} g_{i k_{i}}\left(\bar{\pi}^{*}\right), h\right\rangle \\
& =-N \pi_{i}\left(z_{k_{i}}\right)\left(\sum_{k=1}^{k_{i}-1} \pi_{i}\left(z_{k}\right) h_{k}+\left(\pi_{i}\left(z_{k_{i}}\right)-1\right) h_{k_{i}}\right. \\
& \left.\quad+\sum_{k=k_{i}+1}^{N} \pi_{i}\left(z_{k}\right) h_{k}\right)
\end{aligned}
$$

где $i \in\{1, \ldots, n\}$. Пусть $a_{1}, \ldots, a_{n}<0$. Рассмотрим следующую систему линейных алгебраических уравнений относительно $h_{1}, \ldots, h_{N}$ :

$$
\begin{gathered}
\sum_{k=1}^{k_{i}-1} \pi_{i}\left(z_{k}\right) h_{k}+\left(\pi_{i}\left(z_{k_{i}}\right)-1\right) h_{k_{i}}+\sum_{k=k_{i}+1}^{N} \pi_{i}\left(z_{k}\right) h_{k}=a_{k} \\
(i \in\{1, \ldots, n\}) \\
h_{1}+\cdots+h_{N}=0 .
\end{gathered}
$$

Ее матричная запись имеет вид $A h^{T}=a^{T}$, где матрица $A$ задана формулой $(2.2), a=\left(a_{1}, \ldots, a_{n}, 0\right)$ и $T$ - знак транспонирования для векторовстрок. По предположению, во-первых, ранг матрицы $A$ не меньше, чем $n+1$ - число ее строк (число уравнений в системе $(2.11),(2.12))$, и, вовторых, последнее число не превосходит $N$ - числа столбцов матрицы $A$ (числа неизвестных в системе $(2.11),(2.12))$. Поэтому система уравнений $(2.11),(2.12)$ имеет решение. Пусть $h=\left(h_{1}, \ldots, h_{N}\right)$ - ее решение. Поскольку $a_{1}, \ldots, a_{n}<0$, правые части равенств (2.10) положительны, и, следовательно, неравенства (2.9) имеют место. Доказательство закончено.

\section{3. Алгебраические свойства интеграции.}

3.1. Интеграция как умножение. В следующей теореме фиксируется тот факт, что интеграция как бинарная операция коммутативна и ассоциативна, т.е. обладает характерными алгебраическими свойствами умножения. Сначала приведем следующее очевидное замечание.

3 а м е ч а н и е 3.1. Если $\left(\pi_{1}, \pi_{2}, \pi_{3}\right) \in \Pi^{(3)}$, то $\left(\left(\pi_{1} \cdot \pi_{2}\right), \pi_{3}\right),\left(\pi_{1}\right.$, $\left.\left(\pi_{2}, \pi_{3}\right)\right) \in \Pi^{(2)}$. 
Теорема 3.1. Справедливь следующие утверждения. $\Pi^{(2)}$.

1. Интеграчия коммутативна, т.е. $\pi_{1} \cdot \pi_{2}=\pi_{2} \cdot \pi_{1} \partial л я в$ всех $\left(\pi_{1}, \pi_{2}\right) \in$

2. Интеграция ассоииативна, т.е. $\left(\pi_{1} \cdot \pi_{2}\right) \cdot \pi_{3}=\pi_{1} \cdot\left(\pi_{2} \cdot \pi_{3}\right)$ для $\operatorname{вcex}\left(\pi_{1}, \pi_{2}, \pi_{3}\right) \in \Pi^{(3)}$.

Д о к а з а т е л ь с в о. Утверждение 1 очевидно. Докажем утверждение 2. Возьмем произвольный набор $\left(\pi_{1}, \pi_{2}, \pi_{3}\right) \in \Pi^{(3)}$. Рассмотрим произвольный элемент $z \in Z$. По определению имеем

$$
\left(\pi_{1} \cdot \pi_{2}\right)(z)=\pi_{1}(z) \pi_{2}(z) c_{12}, \quad \text { где } \quad c_{12}=\frac{1}{\sum_{z^{\prime} \in Z} \pi_{1}\left(z^{\prime}\right) \pi_{2}\left(z^{\prime}\right)}
$$

и

$$
\left(\left(\pi_{1} \cdot \pi_{2}\right) \cdot \pi_{3}\right)(z)=\left(\pi_{1} \cdot \pi_{2}\right)(Z) \pi_{3}(z) c_{(12) 3}=\pi_{1}(z) \pi_{2}(z) \pi_{3}(z) c_{12} c_{(12) 3},
$$

где

$$
c_{(12) 3}=\frac{1}{\sum_{z^{\prime} \in Z}\left(\pi_{1} \cdot \pi_{2}\right)\left(z^{\prime}\right) \pi_{3}\left(z^{\prime}\right)}=\frac{1}{\sum_{z^{\prime} \in Z} \pi_{1}\left(z^{\prime}\right) \pi_{2}\left(z^{\prime}\right) \pi_{3}\left(z^{\prime}\right) c_{12}} .
$$

Отсюда получаем, что

$$
\left(\left(\pi_{1} \cdot \pi_{2}\right) \cdot \pi_{3}\right)(z)=\frac{\pi_{1}(z) \pi_{2}(z) \pi_{3}(z)}{\sum_{z^{\prime} \in Z} \pi_{1}\left(z^{\prime}\right) \pi_{2}\left(z^{\prime}\right) \pi_{3}\left(z^{\prime}\right)} .
$$

Аналогично устанавливаем, что правая часть последнего равенства совпадает с $\left(\pi_{1} \cdot\left(\pi_{2} \cdot \pi_{3}\right)\right)(z)$. Ввиду произвольности $z \in Z$ имеем $\left(\pi_{1} \cdot \pi_{2}\right) \cdot \pi_{3}=\pi_{1} \cdot\left(\pi_{2} \cdot \pi_{3}\right)$. Доказательство закончено.

3 а м е ч а н и е 3.2. Замечание 2.1 допускает следующую интерпретацию: относительно интеграции как умножения равномерная вероятность $\bar{\pi}$ играет роль единицы, а каждая концентрированная вероятность - роль нуля.

Из теоремы 3.1 сразу вытекает следующее утверждение.

Следствие 3.1. Для каждого натурального $n>1$ и каждого набора $\left(\pi_{1}, \ldots, \pi_{n}\right) \in \Pi^{(n)}$ результат $\pi_{1} \cdot \ldots \cdot \pi_{n}$ интеграции не меняется при «перемножении»в любом порядке и за любое число шагов, а именно: $\pi_{1} \cdot \ldots \cdot \pi_{n}=\left(\pi_{i_{1}} \cdot \ldots \cdot \pi_{i_{k_{1}}}\right) \cdot\left(\pi_{i_{k_{1}}+1} \cdot \ldots \cdot \pi_{i_{k_{2}}}\right) \cdot \ldots \cdot\left(\pi_{i_{k_{m}}} \cdot \ldots \cdot \pi_{i_{n}}\right)$ для любой перестановки $\left(i_{1}, \ldots, i_{n}\right)$ в $(1, \ldots, n)$ и любой возрастающей последовательности $\left(k_{j}\right)_{1}^{m}$ из $\{2, \ldots, n-1\}$.

3.2. Интеграционные степени. Основываясь на следствии 3.1 , для каждой вероятности $\pi \in \Pi$ и каждого натурального $n>1$ будем обозначать через $\pi^{n}$ результат интеграции $n$ копий $\pi$; вероятность $\pi^{n}$ будем называть $n$-й интеграционной степенью вероятности $\pi$; саму вероятность $\pi$ будем для единообразия называть ее первой интеграционной степенью и обозначать $\pi^{-1}$. 
Следующая теорема, вытекающая непосредственно из определения результата интеграции, фиксирует тот факт, что $n$-я интеграционная степень вероятности наследует упорядоченность ее значений и при росте $n$ углубляет различия между ними.

Теорема 3.2. Для любой вероятности $\pi \in \Pi$ и любого натурального п справедливы следующие утверждения.

1. $Z^{+}\left(\pi^{n}\right)=Z^{+}(\pi)$.

2. Для всяких элементов $z_{1}, z_{2} \in Z^{+}(\pi)$ выполняется равенство

$$
\frac{\pi^{n}\left(z_{1}\right)}{\pi^{n}\left(z_{2}\right)}=\left(\frac{\pi\left(z_{1}\right)}{\pi\left(z_{2}\right)}\right)^{n} .
$$

Теорема 3.2 с очевидностью влечет следующее.

Следствие 3.2. Пусть $Z_{*}$ - множество всех точек максимума вероятности $\pi \in \Pi$ и вероятность $\pi_{*} \in \Pi$ равномерна на $Z_{*}$, m.е. $\pi_{*}(z)=0$ для всех $z \in Z \backslash Z^{+}(\pi) u \pi_{*}(z)=1 /\left|Z_{*}\right|$ для всех $z \in Z_{*}$. Тогда $\pi^{n} \rightarrow \pi_{*}$ в П при $n \rightarrow \infty$.

Вероятность $\pi_{*} \in$ П будем называть интеграционным корнем $n$-й степени их вероятности $\pi \in \Pi$, если $\pi_{*}^{n}=\pi$ (здесь $n$ натуральное). В соответствии с теоремой 3.2 интеграционный корень $\pi_{*} n$-й степени из вероятности $\pi \in \Pi$ наследует упорядоченность значений вероятности $\pi$ и сглаживает различия между ними; это может допускать ту интерпретацию, что оценка $\pi_{*}$ доставляется некоторым несовершенным прототипом метода, стоящего за оценкой $\pi$.

Теорема 3.3. Для каждой вероятности $\pi \in \Pi$ и каждого натурального $n$ существует единственный интеграционный корень $n$-й степени из $\pi$.

Д о к а з а т е л ь с т в о. Рассмотрим какую-либо вероятность $\pi \in$ П. По определению вероятность $\pi_{*} \in$ П есть интеграционный корень $n$-й степени из $\pi$, если $\pi_{*}^{n}=\pi$ или, что то же,

$$
\frac{\pi_{*}^{n}(z)}{\sum_{z^{\prime} \in Z} \pi_{*}^{n}\left(z^{\prime}\right)}=\pi(z) \quad(z \in Z) .
$$

Упорядочим элементы множества $Z$, т.е. положим $Z=\left\{z_{1}, \ldots, z_{N}\right\}$, где $N=|Z|$. Тогда критерий того, что $\pi_{*} \in \Pi$ есть интеграционный корень $n$-й степени из $\pi$, состоит в том, что вектор $\left(\pi_{*}^{n}\left(z_{1}\right), \ldots, \pi_{*}^{n}\left(z_{N}\right), \sum_{i=1}^{N} \pi_{*}^{n}\left(z_{i}\right)\right)$ решает систему

$$
\begin{aligned}
x_{1}-\pi\left(z_{1}\right) x_{N+1} & =0, \\
\ldots \ldots \ldots \cdots \cdots \cdots & \ldots \cdots \\
x_{N}-\pi\left(z_{N}\right) x_{N+1} & =0 \\
x_{1}+\cdots+x_{N}-x_{N+1} & =0
\end{aligned}
$$


алгебраических уравнений при дополнительных ограничениях

$$
x_{1}, \ldots, x_{N} \geqslant 0, \quad x_{1}^{1 / n}+\cdots+x_{N}^{1 / n}=1 .
$$

Пусть $A$ обозначает матрицу систему (3.1). Имеем:

$$
A=\left(\begin{array}{ccccccc}
1 & 0 & 0 & \ldots & 0 & 0 & -\pi\left(z_{1}\right) \\
0 & 1 & 0 & \ldots & 0 & 0 & -\pi\left(z_{2}\right) \\
\ldots & \ldots & \ldots & \ldots & \ldots & \ldots & \ldots \\
0 & 0 & 0 & \ldots & 0 & 1 & -\pi\left(z_{N}\right) \\
1 & 1 & 1 & \ldots & 1 & 1 & -1
\end{array}\right)
$$

Сумма первых $N$ строк матрицы $A$ равна ее $(N+1)$-й строке, и ее левая верхняя $(N \times N)$-подматрица невырождена. Значит, $((N+1) \times(N+1))$ матрица $A$ имеет ранг $N$. Следовательно, множество всех решений системы (3.1) образует одномерное подпространство в $\mathbf{R}^{N+1}$. Пусть $\left(y_{1}, \ldots, y_{N+1}\right)$ - какое-либо ненулевое решение системы (3.1). Поскольку $\pi\left(z_{1}\right), \ldots, \pi\left(z_{N}\right)$ неотрицательны и среди них есть не равные нулю, то, как видно из $(3.1), y_{N+1} \neq 0$, среди чисел $y_{1}, \ldots, y_{N}$ есть отличные от нуля и знаки всех таких отличных от нуля чисел совпадают со знаком числа $y_{N+1}$. Без нарушения общности будем считать, что $y_{N+1}>0$ (в противном случае умножим $y_{1}, \ldots, y_{N+1}$ на -1$)$. Тогда $y_{1}, \ldots, y_{N+1} \geqslant 0$.

Пусть

$$
\begin{gathered}
\lambda=\left(\frac{1}{y_{1}^{1 / n}+\cdots+y_{N}^{1 / N}}\right)^{n}, \\
x_{i}=\lambda y_{i} \quad(i \in\{1, \ldots, N+1\}) .
\end{gathered}
$$

Очевидно, что $x_{1}, \ldots, x_{N}$ удовлетворяют неравенству из (3.2). Далее,

$$
x_{1}^{1 / n}+\cdots+x_{N}^{1 / n}=\lambda^{1 / n}\left(y_{1}^{1 / n}+\cdots+y_{N}^{1 / n}\right)=1 ;
$$

таким образом, для $x_{1}, \ldots, x_{N}$ верно равенство из (3.2). Значит, интеграционный корень $\pi_{*} n$-й степени из $\pi$ существует и находится из соотношений

$$
\pi_{*}\left(z_{i}\right)=x_{i}^{1 / n} \quad(i \in\{1, \ldots, N\}) .
$$

Если $\pi_{*}$ - интеграционный корень $n$-й степени из $\pi$, числа $x_{1}, \ldots, x_{N}$ определены формулой $(3.6)$ и $x_{N+1}=x_{1}+\cdots+x_{N}$, то, как установлено выше, $x_{1}, \ldots, x_{N+1}$ доставляют решение системы (3.1) и удовлетворяют ограничениям (3.2). Поскольку подпространство всех решений системы (3.1) одномерно, при некотором действительном $\lambda$ выполняется (3.4). Тогда (3.2) влечет за собой (3.5); следовательно, $\lambda$ определяется из (3.3). Таким образом, интеграционный корень $n$-й степени из $\pi$ единственен. Доказательство закончено. 
Поскольку, согласно теореме 3.3 , для всего натурального $n$ интеграционный корень $n$-й степени из произвольной вероятности $\pi \in \Pi$ существует и единственен, будем для него использовать обозначение $\pi^{1 / n}$.

3 а м е ч а н и е 3.3. Из теоремы 3.2 следует, что для произвольной вероятности $\pi \in \Pi$ и всякого элемента $z \in Z \backslash Z^{+}(\pi)$ при всех натуральных $n$ выполняется равенство $\pi^{1 / n}(z)=0$ и для всяких элементов $z_{1}, z_{2} \in Z^{+}(\pi)$ имеет место сходимость

$$
\frac{\pi^{1 / n}\left(z_{1}\right)}{\pi^{1 / n}\left(z_{2}\right)}=\left(\frac{\pi\left(z_{1}\right)}{\pi\left(z_{2}\right)}\right)^{1 / n} \rightarrow 1 \quad \text { при } n \rightarrow \infty \text {. }
$$

Последнее означает, что $\pi^{1 / n} \rightarrow \pi_{*}$ в П, где вероятность $\pi_{*}$ равномерна на $Z^{+}(\pi)$, т.е. такова, что $\pi_{*}(z)=0$ для всех $z \in Z \backslash Z^{+}(\pi)$ и $\pi_{*}(z)=$ $1 /\left|Z^{+}(\pi)\right|$ для всех $z \in Z^{+}(\pi)$.

Апеллируя к теореме 3.3 , введем в рассмотрение рациональные интеграционные степени вероятностей. Именно, для всякой вероятности $\pi \in \Pi$ и всяких натуральных $n$ и $m$ вероятность $\pi^{m / n}=\left(\pi^{m}\right)^{1 / n}$ будем называть $m / n$-й интеграиионной степенью вероятности $\pi$.

3 а м е ч а н и е 3.4. Для рациональных интеграционных степеней вероятностей справедливы стандартные арифметические соотношения. Точнее, для каждой вероятности $\pi \in \Pi$ и каждых натуральных $n$ и $m$ вероятность $\pi^{m / n}=\left(\pi^{m}\right)^{1 / n}$ можно также определить, как $\pi^{m / n}=\left(\pi^{1 / n}\right)^{m}$. В самом деле, имеем $\left(\left(\pi^{1 / n}\right)^{m}\right)^{n}=\left(\pi^{1 / n}\right)^{m n}=\left(\pi^{1 / n}\right)^{n m}=\left(\left(\pi^{1 / n}\right)^{n}\right)^{m}=\pi^{m}$. Отсюда, обращаясь к определению корня $n$-й степени из вероятности $\pi^{m}$, получаем, что $\pi^{m / n}=\left(\pi^{1 / n}\right)^{m}$.

3.3. Дезинтеграция. Из определения интеграции вероятностей следует, что $Z^{+}\left(\pi \cdot \pi_{1}\right)=Z^{+}(\pi) \cap Z^{+}\left(\pi_{1}\right)$ для всяких вероятностей $\left(\pi, \pi_{1}\right) \in \Pi^{(2)}$. Имея это в виду, дадим следующее определение. Для вероятностей $\pi_{1}, \pi_{2} \in \Pi$ таких, что $Z^{+}\left(\pi_{2}\right) \subset Z^{+}\left(\pi_{1}\right)$, вероятность $\pi \in \Pi$ будем называть результатом дезинтеграиии вероятности $\pi_{2}$ над вероятностью $\pi_{1}$, если $Z^{+}\left(\pi_{2}\right)=Z^{+}(\pi) \cap Z^{+}\left(\pi_{1}\right)$ (и тогда $\left.\left(\pi, \pi_{1}\right) \in \Pi^{(2)}\right)$ и $\pi \cdot \pi_{1}=\pi_{2}$.

Теорема 3.4. Пусть вероятности $\pi_{1}, \pi_{2} \in \Pi$ таковь, ито $Z^{+}\left(\pi_{2}\right) \subset Z^{+}\left(\pi_{1}\right)$. Справедливь следующие утверждения.

1. Существует результат дезинтеграции вероятности $\pi_{2}$ над вероятностью $\pi_{1}$.

2. Если вероятность $\pi$ есть результат дезинтеграции вероятности $\pi_{2}$ над вероятностью $\pi_{1}$, то вероятность $\pi^{\prime} \in \Pi$ также есть результат дезинтеграчии вероятности $\pi_{2}$ над вероятностью $\pi_{1}$ тогда и только тогда, когда при некотором $\mu>0$ верно равенство $\left.\pi^{\prime}\right|_{Z^{+}\left(\pi_{1}\right)}=\left.\mu \pi\right|_{Z^{+}\left(\pi_{1}\right)}$, где $\left.\left.\pi\right|_{Z^{+}\left(\pi_{1}\right)} u \pi^{\prime}\right|_{Z^{+}\left(\pi_{1}\right)}-$ сужения на $Z^{+}\left(\pi_{1}\right)$ вероятностей $\pi$ и $\pi^{\prime}$ соответственно. 
Д о к а з а т е л ь с т в о. Упорядочим элементы множества $Z$, положив $Z=\left\{z_{1} \ldots, z_{N}\right\}$, где $N=|Z|$, таким образом, что $Z^{+}\left(\pi_{2}\right)=$ $\left\{z_{1}, \ldots, z_{k}\right\}$ и $Z^{+}\left(\pi_{1}\right)=\left\{z_{1}, \ldots, z_{m}\right\}$ при некоторых $k, m \in\{1, \ldots, N\}$, $m \geqslant k$. Тогда

$$
\begin{array}{cl}
\pi_{1}\left(z_{i}\right) \pi_{2}\left(z_{i}\right)<0 & (i \in\{1, \ldots, k\}), \\
\pi_{1}\left(z_{i}\right)<0, \quad \pi_{2}\left(z_{i}\right)=0 & (i \in\{k+1, \ldots, m\}), \\
\pi_{1}, \pi_{2}\left(z_{i}\right)=0 & (i \in\{m+1, \ldots, N\}) .
\end{array}
$$

По определению вероятность $\pi \in \Pi$ есть результат дезинтеграции вероятности $\pi_{2}$ над вероятностью $\pi_{1}$, если $Z^{+}(\pi) \cap Z^{+}\left(\pi_{1}\right)$ и

$$
\frac{\pi(z) \pi_{1}(z)}{\sum_{z^{\prime} \in Z} \pi\left(z^{\prime}\right) \pi_{1}\left(z^{\prime}\right)}=\pi_{2}(z) \quad(z \in Z)
$$

или, равносильно, вектор $\left(\pi\left(z_{1}\right), \ldots, \pi\left(z_{N}\right), \sum_{i=1}^{N} \pi\left(z_{i}\right) \pi_{1}\left(z_{i}\right)\right)$ есть решение системы алгебраических уравнений

$$
\begin{aligned}
& \pi_{1}\left(z_{1}\right) x_{1}-\pi_{2}\left(z_{1}\right) x_{N+1}=0 \\
& \ldots \ldots \ldots \ldots \ldots \ldots \ldots \ldots \ldots \ldots \ldots \\
& \pi_{1}\left(z_{N}\right) x_{N}-\pi_{2}\left(z_{N}\right) x_{N+1}=0 \\
& \pi_{1}\left(z_{1}\right) x_{1}+\cdots+\pi_{1}\left(z_{N}\right) x_{N}-x_{N+1}=0
\end{aligned}
$$

при дополнительных ограничениях

$x_{1}, \ldots, x_{k}>0, \quad x_{k+1}, \ldots, x_{m}=0, \quad x_{m+1}, \ldots, x_{N} \geqslant 0, \quad x_{1}+\cdots+x_{N}=1$

(в случаях $k=m$ и $m=N$ следует опустить соответственно второе и третье ограничение).

При $k<m$ положим

$$
x_{k+1}, \ldots, x_{m}=0 .
$$

Тогда, в силу $(3.8), x_{k+1}, \ldots, x_{m}$ удовлетворяют уравнениям, записанным в $(3.10)$ в строках с номерами $k+1, \ldots, m$. Обращаясь к $(3.9)$, видим, что если $m<N$, то уравнениям, записанным в (3.10) в строках с номерами $m+1, \ldots, N$, удовлетворяют произвольные числа $x_{m+1}, \ldots, x_{N}$.

Рассмотрим оставшуюся часть системы (3.10), т.е. ее подсистему, составленную из уравнений, записанных в строках $1, \ldots, k$ и $N+1$ :

$$
\begin{aligned}
& \pi_{1}\left(z_{1}\right) x_{1}-\pi_{2}\left(z_{1}\right) x_{N+1}=0 \\
& \ldots \ldots \ldots \ldots \ldots \ldots \ldots \ldots \ldots \ldots \\
& \pi_{1}\left(z_{k}\right) x_{k}-\pi_{2}\left(z_{k}\right) x_{N+1}=0 \\
& \pi_{1}\left(z_{1}\right) x_{1}+\cdots+\pi_{1}\left(z_{k}\right) x_{k}-x_{N+1}=0 .
\end{aligned}
$$


Последнее уравнение из (3.13) эквивалентно $(N+1)$-му уравнению из (3.10); это следует из (3.12) и (3.9). Пусть $A$ обозначает матрицу системы (3.13). Имеем

$$
A=\left(\begin{array}{ccccccc}
\pi_{1}\left(z_{1}\right) & 0 & 0 & \ldots & 0 & 0 & -\pi_{2}\left(z_{1}\right) \\
0 & \pi_{1}\left(z_{2}\right) & 0 & \ldots & 0 & 0 & -\pi_{2}\left(z_{2}\right) \\
\ldots & \ldots & \ldots & \ldots & \ldots & \ldots & \ldots \\
0 & 0 & 0 \ldots & 0 & -\pi_{2}\left(z_{1}\right) & -\pi_{2}\left(z_{k}\right) & \\
\pi_{1}\left(z_{1}\right) & \pi_{1}\left(z_{2}\right) & \pi_{1}\left(z_{3}\right) & \ldots & \pi_{1}\left(z_{k-2}\right) & \pi_{1}\left(z_{k-2}\right) & -1
\end{array}\right) .
$$

Первые $k$ строк матрицы $A$ в сумме дают ее $(k+1)$-ю строку, а ее верхняя левая $(k \times k)$-подматрица невырождена ввиду (3.7). Значит, ранг $((k+1) \times(k+1))$-матрицы $A$ равен $k$. Следовательно, множество всех решений системы (3.13) есть одномерное пространство в $\mathbf{R}^{k+1}$. Пусть $\left(y_{1}, \ldots, y_{k}, y_{N+1}\right)$ - ненулевое решение системы (3.13). Очевидно, что $y_{N+1} \neq 0$. Без потери общности будем считать, что $y_{N+1}>0$ (в противном случае умножим $\left(y_{1}, \ldots, y_{k}, y_{N+1}\right)$ на -1$)$. Тогда $y_{1}, \ldots, y_{k}, y_{N+1}>0$. Возьмем $c \in(0,1]$ и положим

$$
\lambda=\frac{c}{y_{1}+\cdots+y_{k}}, \quad x_{i}=\lambda y_{i} \quad(i \in\{1, \ldots, k, N+1\}) .
$$

Ясно, что $x_{1}, \ldots, x_{k}>0, x_{1}+\cdots+x_{k}=c$. Объединяя это с $(3.12)$, получаем, что $x_{1}, \ldots, x_{m}$ удовлетворяют первому и последнему ограничениям из (3.11).

Если $m=N$, то, полагая $c=1$ и (в случае $m>k$ ) обращаясь к $(3.12)$, получаем, что набор $\left(x_{1}, \ldots, x_{N}, x_{N+1}\right)$ неотрицательных чисел решает систему (3.10) и при этом удовлетворяет ограничениям (3.11) (где третье ограничение следует опустить, а второе следует опустить в случае $m=k$ ). Если $m<N$, возьмем произвольные $x_{m+1}, \ldots, x_{N} \geqslant 0$, дающие в сумме $1-c$. Видим, что набор $\left(x_{1}, \ldots, x_{N}, x_{N+1}\right)$ решает систему (3.10) и при этом удовлетворяет ограничениям (3.11) (где второе ограничение следует опустить при $m=k$ ). Таким образом, вероятность $\pi \in \Pi$, определяемая соотношениями

$$
\pi\left(z_{i}\right)=x_{i} \quad(i \in\{1, \ldots, N\})
$$

есть результат дезинтеграции вероятности $\pi_{2}$ над вероятностью $\pi_{1}$. У тверждение 1 доказано.

Докажем утверждение 2. Пусть вероятность $\pi^{\prime} \in \Pi$ такова, что $\left.\pi^{\prime}\right|_{Z^{+}\left(\pi_{1}\right)}=\left.\mu \pi\right|_{Z^{+}\left(\pi_{1}\right)}$ при некотором $\mu>0$ или, что то же, для ее значений

$$
x_{i}^{\prime}=\pi^{\prime}\left(z_{i}\right) \quad(i \in\{1, \ldots, N\})
$$

выполняются равенства

$$
x_{i}^{\prime}=\mu x_{i} \quad(i \in\{1, \ldots, m\}) .
$$


Тогда, ввиду (3.12) (в случае $m>k$ ), имеем

$$
x_{k+1}^{\prime}, \ldots, x_{m}^{\prime}=0 \text {. }
$$

Пусть

$$
x_{N+1}^{\prime}=\pi_{1}\left(z_{1}\right) x_{1}^{\prime}+\cdots+\pi_{1}\left(z_{N}\right) x_{N}^{\prime} .
$$

Очевидно, что $x_{N+1}^{\prime}=\mu x_{N+1}$. Поскольку набор $\left(x_{1}, \ldots, x_{m}, x_{m+1}^{\prime \prime}, \ldots\right.$, $\left.x_{N}^{\prime \prime}, x_{N+1}\right)$ с произвольными $x_{m+1}^{\prime \prime}, \ldots, x_{N}^{\prime \prime} \geqslant 0$ (при $m<N$ ) peшает систему (3.10) при ограничениях (3.11), получаем, что набор $\left(\pi^{\prime}\left(z_{1}\right), \ldots, \pi^{\prime}\left(z_{N}\right), x_{N+1}^{\prime}\right)=\left(x_{1}^{\prime}, \ldots, x_{N+1}^{\prime}\right)$ обладает теми же свойствами. Следовательно, вероятность $\pi$ есть результат дезинтеграции вероятности $\pi_{2}$ над вероятностью $\pi_{1}$.

Обратно, пусть вероятность $\pi^{\prime}$ есть результат дезинтеграции вероятности $\pi_{2}$ над вероятностью $\pi_{1}$. Тогда набор $\left(x_{1}^{\prime}, \ldots, x_{N+1}^{\prime}\right)$, определенный формулами (3.16) и (3.18), есть решение системы (3.10), удовлетворяющее ограничениям (3.11); в частности, при $m>k$ имеем (3.17). Тогда

$$
x_{i}^{\prime}=\lambda y_{i} \quad(i \in\{1, \ldots, k, N+1\})
$$

при некотором $\lambda^{\prime}>0$. Поэтому согласно (3.14)

$$
x_{i}^{\prime}=\mu x_{i} \quad(i \in\{1, \ldots, k, N+1\}),
$$

где $\mu=\lambda^{\prime} / \lambda$. Значит, ввиду (3.15), (3.16), (3.12) и (3.17) (последние два соотношения имеют место лишь в случае $m>k$ ), получаем, что

$$
\pi^{\prime}\left(z_{i}\right)=\mu \pi\left(z_{i}\right) \quad(i \in\{1, \ldots, m\}) .
$$

Следовательно, $\left.\pi^{\prime}\right|_{Z^{+}\left(\pi_{1}\right)}=\left.\mu \pi\right|_{Z^{+}\left(\pi_{1}\right)}$. Теорема 3.4 доказана.

Для произвольных вероятностей $\pi_{1}, \pi_{2} \in \Pi$ таких, что $Z^{+}\left(\pi_{2}\right) \subset$ $Z^{+}\left(\pi_{1}\right)$, будем обозначать через $\left[\pi_{2} / \pi_{1}\right]$ множество всех результатов дезинтеграции вероятности $\pi_{2}$ над вероятностью $\pi_{1}$. Многозначное отображение $\left(\pi_{1}, \pi_{2}\right) \mapsto\left[\pi_{2} / \pi_{1}\right]$, определенное на множестве всех $\left(\pi_{1}, \pi_{2}\right) \in \Pi^{2}$ таких, что $Z^{+}\left(\pi_{2}\right) \subset Z^{+}\left(\pi_{1}\right)$, будем называть дезинтеграцией.

Следствие 3.3. Пусть вероятности $\pi_{1}, \pi_{2} \in \Pi$ maковь, что $Z^{+}\left(\pi_{2}\right) \subset Z^{+}\left(\pi_{1}\right)$. Справедливь следующие утверждения.

1. Если $Z^{+}\left(\pi_{1}\right)=Z$, то множество $\left[\pi_{2} / \pi_{1}\right]$ одноэлементно.

2. Eсли $Z^{+}\left(\pi_{1}\right) \neq Z$ u $\pi \in\left[\pi_{2} / \pi_{1}\right]$, mo

$$
\left[\pi_{2} / \pi_{1}\right]=\left\{\pi^{\prime} \in \Pi:\left.\pi^{\prime}\right|_{Z^{+}\left(\pi_{1}\right)}=\left.\mu \pi\right|_{Z^{+}\left(\pi_{1}\right)}, 0<\mu \leqslant \frac{1}{\sum_{z \in Z^{+}\left(\pi_{1}\right)} \pi(z)}\right\} .
$$

Д ок аз а т е л с т в о. Пусть $Z^{+}\left(\pi_{1}\right)=Z$. Если $\pi^{\prime} \in\left[\pi_{2} / \pi_{1}\right]$, то по утверждению 2 теоремы 3.4 имеем $\pi^{\prime}=\left.\pi^{\prime}\right|_{Z^{+}\left(\pi_{1}\right)}=\left.\mu \pi\right|_{Z^{+}\left(\pi_{1}\right)}=\mu \pi$ при 
некотором $\mu>0$. Поскольку $\pi, \pi^{\prime} \in \Pi$, необходимо $\mu=1$. Утверждение 1 доказано.

Пусть $Z^{+}\left(\pi_{1}\right) \neq Z$. По утверждению 2 теоремы 3.4 включение $\pi^{\prime} \in$ $\left[\pi_{2} / \pi_{1}\right]$ имеет место тогда и только тогда, когда $\left.\pi^{\prime}\right|_{Z^{+}\left(\pi_{1}\right)}=\left.\mu \pi\right|_{Z^{+}\left(\pi_{1}\right)}$ при некотором $\mu>0$. Последнее, в свою очередь, возможно тогда и только тогда, когда $\mu \sum_{z \in Z^{+}\left(\pi_{1}\right)} \pi(Z) \in(0,1]$. У тверждение 2 доказано.

3 а м е ч а н и е 3.5. В предположениях следствия 3.3 существует единственная вероятность $\pi^{\prime} \in\left[\pi_{2} / \pi_{1}\right]$ такая, что $Z^{+}\left(\pi^{\prime}\right)=Z^{+}\left(\pi_{2}\right)$. В самом деле, пусть вероятность $\pi^{\prime} \in\left[\pi_{2} / \pi_{1}\right]$ такова, что

$$
\left.\pi^{\prime}\right|_{Z^{+}\left(\pi_{1}\right)}=\left.\frac{1}{\sum_{z \in Z^{+}\left(\pi_{1}\right)} \pi(z)} \pi\right|_{Z^{+}\left(\pi_{1}\right)} .
$$

Тогда $\sum_{z \in Z^{+}\left(\pi_{1}\right)} \pi^{\prime}(z)=1$, откуда следует, что $Z^{+}\left(\pi^{\prime}\right) \subset Z^{+}\left(\pi_{1}\right)$. Далее, тот факт, что $\pi^{\prime} \cdot \pi_{1}=\pi_{2}$, влечет, что $\pi^{\prime}(z)=0$ для каждого $z \in Z^{+}\left(\pi_{1}\right) \backslash$ $Z^{+}\left(\pi_{2}\right)$ и $\pi^{\prime}(z)>0$ для каждого $z \in Z^{+}\left(\pi_{2}\right)$. Следовательно, $Z^{+}\left(\pi^{\prime}\right)=$ $Z^{+}\left(\pi_{2}\right)$. Обратно, если вероятность $\pi^{\prime} \in\left[\pi_{2} / \pi_{1}\right]$ такова, что $Z^{+}\left(\pi^{\prime}\right)=$ $Z^{+}\left(\pi_{2}\right)$, то с необходимостью выполняется (3.19).

Исходя из замечания 3.5 , для любых вероятностей $\pi_{1}, \pi_{2} \in \Pi$ таких, что $Z^{+}\left(\pi_{2}\right) \subset Z^{+}\left(\pi_{1}\right)$, будем обозначать через $\pi_{2} / \pi_{1}$ единственную вероятность $\pi \in\left[\pi_{2} / \pi_{1}\right]$ такую, что $Z^{+}(\pi)=Z^{+}\left(\pi_{2}\right)$.

3 а м е ч а н и е 3.6. Для каждой вероятности $\pi \in \Pi^{+}$(с положительными значениями, см. обозначения в разделе 1$)$ имеем $Z^{+}(\pi)=Z$. Поэтому сужение дезинтеграции на произведение $\Pi^{+} \times \Pi^{+}$определено корректно. По утверждению 1 следствия 3.3 сужение дезинтеграции на произведение $\Pi^{+} \times \Pi^{+}$есть однозначное отображение; оно с очевидностью принимает значения в множестве $\Pi^{+}$. Таким образом, множество $\Pi^{+}$ инвариантно как относительно интеграции, так и относительно дезинтеграции. Легко видеть, что дезинтеграция как (однозначная) функция на $\Pi^{+} \times \Pi^{+}$со значениями в $\Pi^{+}$непрерывна. Как было отмечено ранее (см. замечание 1.1), интеграция также непрерывна. Следовательно, с учетом коммутативности и ассоциативности интеграции (теорема 3.1 ), заключаем, что множество $\Pi^{+}$, оснащенное интеграцией, понимаемой как алгебраическая операция умножения, есть абелева топологическая группа, в которой роль единицы выполняет равномерная вероятность $\bar{\pi}$ (см. замечание 2.1).

По отношению к операции интеграции операция дезинтеграции играет ту же роль, которую в арифметике операция деления играет по отношению к операции умножения. Ограничение $Z^{+}\left(\pi_{2}\right) \subset Z^{+}\left(\pi_{1}\right)$ при определении «частного» $\left[\pi_{2} / \pi_{1}\right]$ есть аналог стандартного арифметического ограничения не обращения в нуль делителя. Следующая теорема констатирует, что отношения между операциями интеграции и дезинтеграции совершенно аналогичны отношениям между арифметическими операциями умножения и деления. 
Теорема 3.5. Справедливы следующие утверждения.

1. Пусть вероятности $\pi_{1}, \pi_{2}, \pi_{3} \in \Pi$ таковы, что $Z^{+}\left(\pi_{3}\right) \subset$ $Z^{+}\left(\pi_{2}\right) \subset Z^{+}\left(\pi_{1}\right)$. Тогда

$$
\left[\left[\pi_{3} / \pi_{2}\right] / \pi_{1}\right]=\left[\pi_{3} /\left(\pi_{2} \cdot \pi_{1}\right)\right]
$$

$2 \partial e$

$$
\left[\left[\pi_{3} / \pi_{2}\right] / \pi_{1}\right]=\bigcup_{\pi^{\prime} \in\left[\pi_{3} / \pi_{2}\right]}\left[\pi^{\prime} / \pi_{1}\right]
$$

2. Пусть вероятности $\pi_{1}, \pi_{2}, \pi_{3} \in \Pi$ таковь, что $Z^{+}\left(\pi_{1}\right)=$ $Z^{+}\left(\pi_{2}\right)=Z^{+}\left(\pi_{3}\right), u \pi_{1} \cdot \pi_{2}=\pi_{1} \cdot \pi_{3}$. Тогда $\pi_{2}=\pi_{3}$.

3. Пусть вероятности $\pi_{1}, \pi_{2}, \pi_{3} \in \Pi$ таковь, что $Z^{+}\left(\pi_{1}\right)=$ $Z^{+}\left(\pi_{2}\right)=Z^{+}\left(\pi_{3}\right)$. Тогда

$$
\left(\pi_{3} \cdot \pi_{2}\right) / \pi_{1}=\left(\pi_{3} / \pi_{1}\right) \cdot \pi_{2}
$$

Д о к а з а т е л с т в о. Докажем утверждение 1. Пусть вероятности $\pi_{1}, \pi_{2}, \pi_{3} \in \Pi$ таковы, что $Z^{+}\left(\pi_{3}\right) \subset Z^{+}\left(\pi_{2}\right) \subset Z^{+}\left(\pi_{1}\right)$. Возьмем какую-либо вероятность $\pi$ из $\left[\left[\pi_{3} / \pi_{2}\right] / \pi_{1}\right]$. Тогда $\pi \in\left[\pi^{\prime} / \pi_{1}\right]$ для некоторого $\pi^{\prime} \in\left[\pi_{3} / \pi_{2}\right]$. Отсюда $\pi \cdot \pi_{1}=\pi^{\prime}$, и $\pi^{\prime} \cdot \pi_{2}=p_{3}$. Поэтому $\pi \cdot \pi_{1} \cdot \pi_{2}=\pi_{3}$ или, что то же, $\pi \cdot\left(\pi_{1} \cdot \pi_{2}\right)=\pi_{3}$. Следовательно, $\pi \in\left[\pi_{3} /\left(\pi_{2} \cdot \pi_{1}\right)\right]$. Мы показатели, что множество, записанное в его правой части. Установим обратное вложение. Возьмем какую-либо вероятность $\pi \in\left[\pi_{3} /\left(\pi_{2} \cdot \pi_{1}\right)\right]$. Имеем $\pi \cdot\left(\pi_{2} \cdot \pi_{1}\right)=\pi_{3}$ или, что то же, $\left(\pi \cdot \pi_{1}\right) \cdot \pi_{2}=\pi_{3}$. Следовательно, $\pi^{\prime}=\left(\pi \cdot \pi_{1}\right) \in\left[\pi_{3} / \pi_{2}\right]$, и $\pi \in\left[\pi^{\prime} / \pi_{1}\right]$. Нужное обратное вложение установлено. Равенство (3.20) справедливо.

Докажем утверждение 2 . Пусть вероятности $\pi_{1}, \pi_{2}, \pi_{3} \in$ П таковы, что $Z^{+}\left(\pi_{3}\right)=Z^{+}\left(\pi_{2}\right)=Z^{+}\left(\pi_{1}\right)$, и $\pi_{1} \cdot \pi_{2}=\pi_{1} \cdot \pi_{3}$. Очевидно, что $\pi_{2}=$ $\left(\pi_{1} \cdot \pi_{2}\right) / \pi_{1}$ и $\pi_{3}=\left(\pi_{1} \cdot \pi_{3}\right) / \pi_{1}$. Отсюда следует, что $\pi_{2}=\pi_{3}$.

Докажем утверждение 3 . Пусть вероятности $\pi_{1}, \pi_{2}, \pi_{3} \in \Pi$ таковы, что $Z^{+}\left(\pi_{1}\right)=Z^{+}\left(\pi_{2}\right)=Z^{+}\left(\pi_{3}\right)$. Положим $\pi=\left(\pi_{3} \cdot \pi_{2}\right) / \pi_{1}$. По определению и по предположению имеем

$$
Z^{+}\left(\pi_{3} \cdot \pi_{2}\right)=Z^{+}\left(\pi_{1}\right)=Z^{+}(\pi)
$$

и $\pi \cdot \pi_{1}=\pi_{3} \cdot \pi_{2}$. Пусть $\pi^{\prime}=\pi / \pi_{2}$. По определению и ввиду (3.22) верны равенства

$$
\begin{gathered}
Z^{+}\left(\pi^{\prime}\right)=Z^{+}(\pi)=Z^{+}\left(\pi_{1}\right), \\
\pi=\pi^{\prime} \cdot \pi_{2}
\end{gathered}
$$

Отсюда $\pi^{\prime} \cdot \pi_{2} \cdot \pi_{1}=\pi_{3} \cdot \pi_{2}$ или

$$
\left(\pi^{\prime} \cdot \pi_{1}\right) \cdot \pi_{2}=\pi_{3} \cdot \pi_{2}
$$


Используя (3.23) и предположение, приходим к равенствам $Z^{+}\left(\pi^{\prime} \cdot \pi_{1}\right)=$ $Z^{+}\left(\pi_{1}\right)=Z^{+}\left(\pi_{2}\right)=Z^{+}\left(\pi_{3}\right)$. Тогда по утверждению 2 из (3.25) следует, что $\pi^{\prime} \cdot \pi_{1}=\pi_{3}$. Значит, $\pi^{\prime}=\pi_{3} / \pi_{1}$. Следовательно, ввиду (3.24) верны равенства $\pi=\pi^{\prime} \cdot \pi_{2}=\left(\pi_{3} / \pi_{1}\right) \cdot \pi_{2}$. Этим доказано равенство (3.21). Доказательство закончено.

\section{4. Интеграция и последовательности вероятностей.}

4.1. Интеграционные пределы. Будем называть последовательность $\left(\pi_{i}\right)_{i=1}^{\infty}$ вероятностей из П непротиворечивой, если для любого натурального $n$ вероятности $\pi_{1}, \ldots, \pi_{n}$ непротиворечивы или, что то же, $\left(\pi_{1}, \ldots, \pi_{n}\right) \in \Pi^{(n)}$. Отметим, что для непротиворечивой последовательности $\left(\pi_{i}\right)_{i=1}^{\infty}$ вероятностей из П результат $\pi_{1} \cdot \ldots \cdot \pi_{n}$ интеграции вероятностей $\pi_{1}, \ldots, \pi_{n}$ определен при любом натуральном $n$. Для произвольной непротиворечивой последовательности $\left(\pi_{i}\right)_{n=1}^{\infty}$ вероятностей из П каждый частичный предел в П последовательности $\left(\pi_{1} \cdot \ldots \cdot \pi_{n}\right)_{n=1}^{\infty}$ будем называть частичным интеграчионным пределом последовательности $\left(\pi_{i}\right)_{i=1}^{\infty}$; если частичный интеграционный предел последовательности $\left(\pi_{i}\right)_{i=1}^{\infty}$ единственен, будем называть его интеграчионным пределом последовательности $\left(\pi_{i}\right)_{i=1}^{\infty}$.

3 а м е ч а н и е 4.1. Как отмечено в разделе 1 , множество П как топологическое пространство есть компакт. Поэтому каждая непротиворечивая последовательность вероятностей из П имеет по крайней мере один частичный предел.

Про непротиворечивую последовательность вероятностей из П будем говорить, что она интеграционно сходится, если она имеет интеграционный предел, и интеграчионно расходится в противном случае. Интеграционно сходящуюся последовательность вероятностей из П будем называть интеграционно сосредоточенной, если ее интеграционный предел есть сосредоточенная вероятность.

Приведенные определения допускают простые содержательные интерпретации. Если последовательность $\left(\pi_{i}\right)_{i=1}^{\infty}$ вероятностей интеграционно сосредоточена, стоящие за ними методы $1,2, \ldots$ наблюдения, взаимодействуя при последовательном добавлении, улучшают информацию о наблюдаемом элементе и в пределе дают полную информацию о нем. Если $\left(\pi_{i}\right)_{i=1}^{\infty}$ интеграционно сходится, но не является интеграционно сосредоточенной, методы $1,2, \ldots$ при последовательной интеграции «находят консенсус» и в пределе доставляют вполне определенную, хотя и неполную, информацию о наблюдаемом элементе. Если $\left(\pi_{i}\right)_{i=1}^{\infty}$ интеграционно расходится, методы $1,2, \ldots$ не доставляют в пределе согласованной информации о наблюдаемом элементе.

Приведем пример непротиворечивой интеграционно расходящейся последовательности вероятностей.

П р и м е р 4.1. Пусть $Z=\left\{z_{1}, z_{2}\right\}$, вероятности $\pi^{(1)}, \pi^{(2)} \in \Pi$ та- 
ковы, что

$$
\pi^{(1)}\left(z_{1}\right)>\pi^{(1)}\left(z_{2}\right)>0, \quad \pi^{(2)}\left(z_{2}\right)>\pi^{(2)}\left(z_{1}\right)>0
$$

и последовательность $\left(\pi_{i}\right)_{i=1}^{\infty}$ вероятностей из П определена следующими условиями:

$$
\begin{aligned}
& \pi_{i}=\pi^{(1)} \quad\left(i \in\left\{1, \ldots, k_{2^{j}-1}\right\}, j \in\{1,2, \ldots\}\right) \\
& \pi_{i}=\pi^{(2)} \quad\left(i \in\left\{k_{2^{j}-1}+1, \ldots, k_{2^{j}}\right\}, j \in\{1,2, \ldots\}\right),
\end{aligned}
$$

где $1<k_{1}<k_{2}<k_{3}<\cdots$. Ясно, что последовательность $\left(\pi_{i}\right)_{i=1}^{\infty}$ непротиворечива. Пусть

$$
\begin{gathered}
\pi^{*} j=\pi_{1} \cdot \ldots \cdot \pi_{k_{j}}, \quad \pi_{j, j+1}^{*}=\pi_{k_{j}+1} \cdot \ldots \cdot \pi_{k_{j+1}} \quad(j=1,2, \ldots), \\
q^{(1)}=\frac{\pi^{(1)}\left(z_{2}\right)}{\pi^{(1)}\left(z_{1}\right)}, \quad q^{(2)}=\frac{\pi^{(2)}\left(z_{2}\right)}{\pi^{(2)}\left(z_{1}\right)} .
\end{gathered}
$$

Очевидно, что $q^{(1)}<1, a^{(2)}>1$. Имеем

$$
\pi_{1}^{*}\left(z_{1}\right)=\frac{\pi^{(1) k_{1}}\left(z_{1}\right)}{\pi^{(1) k_{1}}\left(z_{1}\right)+\pi^{(1) k_{1}}\left(z_{2}\right)}=\frac{1}{1+q^{(1) k_{1}}} .
$$

Возьмем последовательность $\left(\varepsilon_{j}\right)_{j=1}^{\infty}$ положительных чисел, сходящихся к нулю. Пусть натуральное $k_{1}$ таково, что $q^{(1) k_{1}}<\varepsilon_{1}$. Тогда

$$
\pi_{1}^{*}\left(z_{1}\right)>\frac{1}{1+\varepsilon_{1}} .
$$

Далее,

$$
\begin{aligned}
\pi_{1,2}^{*}\left(z_{1}\right) & =\frac{\pi^{(2)\left(k_{2}-k_{1}\right)}\left(z_{1}\right)}{\pi^{(2)\left(k_{2}-k_{1}\right)}\left(z_{1}\right)+\pi^{(2)\left(k_{2}-k_{1}\right)}\left(z_{2}\right)}=\frac{1}{1+q^{(2)\left(k_{2}-k_{1}\right)}}, \\
\pi_{2}^{*}\left(z_{1}\right) & =\left(\pi_{1}^{*} \cdot \pi_{1,2}^{*}\right)\left(z_{1}\right)=\frac{\pi_{1}^{*}\left(z_{1}\right) \pi_{1,2}^{*}\left(z_{1}\right)}{\pi_{1}^{*}\left(z_{1}\right) \pi_{1,2}^{*}\left(z_{1}\right)+\pi_{1}^{*}\left(z_{2}\right) \pi_{1,2}^{*}\left(z_{2}\right)} \\
& <\frac{\pi_{1}^{*}\left(z_{1}\right) \pi_{1,2}^{*}\left(z_{1}\right)}{\pi_{1}^{*}\left(z_{2}\right) \pi_{1,2}^{*}\left(z_{2}\right)}=\frac{\pi_{1}^{*}\left(z_{1}\right)}{\pi_{1}^{*}\left(z_{2}\right)} \frac{\pi_{1,2}^{*}\left(z_{1}\right)}{1-\pi_{1,2}^{*}\left(z_{1}\right)} .
\end{aligned}
$$

Принимая во внимание (4.3) и неравенство $q^{(2)}>1$, выберем натуральное $k_{2}>k_{1}$ так, чтобы правая часть (4.4) была меньше $\varepsilon_{2}$. Тогда

$$
\pi_{2}^{*}\left(z_{1}\right)<\varepsilon_{2}
$$

Так же, как при обеспечении (4.2) и (4.5), выбором натуральных $k_{3}, k_{4}, \ldots$ обеспечим выполнение неравенств

$$
\pi_{2^{j}-1}^{*}\left(z_{1}\right)>\frac{1}{1+\varepsilon_{2^{j}-1}}, \quad \pi_{2^{j}}^{*}\left(z_{1}\right)<\varepsilon_{2^{j}} \quad(j \in\{1,2, \ldots\}) .
$$


Видим, что вероятность $\lim _{j \rightarrow \infty} \pi_{2^{j}-1}^{*}$ сосредоточена в $z_{1}$, а вероятность $\lim _{j \rightarrow \infty} \pi_{2^{j}}^{*}$ сосредоточена в $z_{2}$. Согласно (4.1) обе эти вероятности есть частичные интеграционные пределы последовательности $\left(\pi_{i}\right)_{i=1}^{\infty}$. Следовательно, последовательность $\left(\pi_{i}\right)_{i=1}^{\infty}$ интегрально расходится.

Следующие ниже теоремы 4.1, 4.2 и 4.3 указывают простые условия интеграционной сосредоточенности последовательностей вероятностей. Теорема 4.1 следует непосредственно из того, что сосредоточенные вероятности играют роль нулей относительно интеграции понимаемой как умножение (см. замечание 3.2 ).

Теорема 4.1. Пусть $\left(\pi_{i}\right)_{i=1}^{\infty}$ - непротиворечивая последовательность вероятностей из П и для некоторого натурального $k$ вероятность $\pi_{k}$ сосредоточена. Тогда последовательность $\left(\pi_{i}\right)_{i=1}^{\infty}$ интеграционно сосредоточена $и \pi_{k}$ - ее интеграчионньй предел.

Следующая теорема, вытекающая из определения меры сосредоточенности, доставляет критерий сосредоточенности последовательности вероятностей.

Теорема 4.2. Пусть $\mu$-мера сосредоточенности. Непротиворечивая последовательность $\left(\pi_{i}\right)_{i=1}^{\infty}$ вероятностей из П интеграчионно сосредоточена тогда и только тогда, когда $\lim _{n \rightarrow \infty} \mu\left(\pi_{1} \cdot \ldots \cdot \pi_{n}\right)=1$.

Следующая теорема говорит о том, что если вероятности, составляющие непротиворечивую последовательность, отдают однозначное предпочтение одному и тому же элементу, то последовательность интеграционно сосредоточена в этом элементе.

Теорема 4.3. Пусть последовательность $\left(\pi_{i}\right)_{i=1}^{\infty}$ вероятностей из П непротиворечива и существуют элемент $z_{*} \in Z$ и положительное $q<1$ такие, что $\pi_{i}(z) / \pi_{i}\left(z_{*}\right)<q$ для всех $z \in Z \backslash\left\{z_{*}\right\}$ и всех натуральньх $i$. Тогда последовательность $\left(\pi_{i}\right)_{i=1}^{\infty}$ интеграционно сосредоточена и ее интеграчионный предел сосредоточен в $z_{*}$.

Д о к а з а т е л с с в о. Для каждого элемента $z \in Z$ и каждого натурального $n$ пусть

$$
v_{n}(z)=\frac{\left(\pi_{1} \cdot \ldots \cdot \pi_{n}\right)(z)}{\left(\pi_{1} \cdot \ldots \cdot \pi_{n}\right)\left(z_{*}\right)}=\frac{\pi_{1}(z) \cdots \pi_{n}(z)}{\pi_{1}\left(z_{*}\right) \cdots \pi_{n}\left(z_{*}\right)} .
$$

Для каждого $z \in Z \backslash\left\{z_{*}\right\}$, очевидно, имеем $v_{n}(z) \leqslant q^{n}(n \in\{1,2, \ldots\})$, и, значит, $\left(\pi_{1} \cdot \ldots \cdot \pi_{n}\right)(z) \rightarrow 0$ при $n \rightarrow \infty$. Следовательно, последовательность $\left(\pi_{1} \cdot \ldots \cdot \pi_{n}\right)_{n=1}^{\infty}$ сходится в П к вероятности, сосредоточенной в $z_{*}$. Доказательство закончено.

Следующий пример показывает, что если условия теоремы 4.3 выполнены с $q=1$, то утверждение теоремы, вообще говоря, не имеет места.

П р и м е р 4.2. Пусть $Z=\left\{z, z_{*}\right\}, \pi_{1}, \pi_{2}, \ldots \in$ П и $q_{i}=$ $\pi_{i}(z) / \pi_{i}\left(z_{*}\right) \in(0,1)$ для всех натуральных $i$. Пусть, далее, $q_{i+1}>q_{i}$ 
для всех натуральных $i$ и ряд $\sum_{i=1}^{\infty}\left|\ln q_{i}\right|$ сходится. Тогда последовательность $\left(v_{n}\right)_{n=1}^{\infty}$, определенная формулой $(4.31)$, принимает значения в $(0,1)$ и убывает; следовательно, $v_{n} \rightarrow v \in[0,1)$ при $n \rightarrow \infty$. Значит, последовательность $\left(\pi_{i}\right)_{i=1}^{\infty}$ непротиворечива и интеграционно сходится. Поскольку ряд $\sum_{i=1}^{\infty}\left|\ln q_{i}\right|$ сходится, $v=\lim _{n \rightarrow \infty} q_{1} \cdots q_{n}>0$. Следовательно, интеграционный предел последовательности $\left(\pi_{i}\right)_{i=1}^{\infty}$ не сосредоточен.

Приведем некоторые достаточные условия интеграционной сходимости последовательностей вероятностей. Следующее утверждение вытекает непосредственно из определения интеграции и ее непрерывности (см. замечание 1.1).

Теорема 4.4. Пусть последовательность $\left(\pi_{i}\right)_{i=1}^{\infty}$ вероятностей из П непротиворечива, существует натуральное $k \geqslant 2$ такое, что последовательность $\left(\pi_{i}\right)_{i=1}^{\infty}$ интеграиионно сходится, $и \pi$ - ее интеграчионный предел. Тогда последовательность $\left(\pi_{i}\right)_{i=1}^{\infty}$ интеграчионно сходится и ее интеграционньй предел есть $\pi_{1} \cdot \ldots \cdot \pi_{k-1} \pi$.

Следующая теорема обобщает теорему 4.3 , ее доказательство подобно доказательству последней теоремы.

Теорема 4.5. Пусть последовательность $\left(\pi_{i}\right)_{i=1}^{\infty}$ вероятностей из П непротиворечива, вероятности $\pi_{1}, \pi_{2}, \ldots$ имеют общее множество $Z_{*}$ точек максимума и существует положительное $q<1$ такое, что $\pi_{i}(z) / \pi_{i}\left(z_{*}\right)<q$ для всех $z \in Z \backslash Z_{*}$, всех $z_{*} \in Z_{*}$ и всех натуральньх $i$. Тогда последовательность $\left(\pi_{i}\right)_{i=1}^{\infty}$ интеграционно сходится и ее интеграционный предел $\pi$ есть вероятность, равномерная на $Z_{*}$, т.е. такая, что $\pi(z)=1 /\left|Z_{*}\right|$ для всех $z_{*} \in Z_{*} u \pi(z)=0$ для всех $z \in Z \backslash Z_{*}$.

Следующая теорема утверждает, что последовательность вероятностей интеграционно сходится, если образующие ее вероятности одинаково упорядочивают элементарные события.

Теорема 4.6. Пусть последовательность $\left(\pi_{i}\right)_{i=1}^{\infty}$ вероятностей из П непротиворечива и существует последовательность $\left(z_{k}\right)_{k=1}^{N}$ из $Z$ такая, ито $\left\{z_{1}, \ldots, z_{N}\right\}=Z u \pi_{i}\left(z_{1}\right) \leqslant \cdots \leqslant \pi_{i}\left(z_{N}\right)$ для всех натуральных $i$. Тогда справедливы следующие утверждения.

1. Последовательность $\left(\pi_{i}\right)_{i=1}^{\infty}$ интеграчионно сходится, и для ее интеграционного предела $\pi$ выполняются неравенства $\pi\left(z_{1}\right) \leqslant \cdots \leqslant$ $\pi\left(z_{N}\right)$.

2. Если существуют положительное $q<1$, натуральное $l \in$ $\{1, \ldots, N-1\}$ и подпоследовательность $\left(\pi_{i_{m}}\right)_{m=1}^{\infty}$ последовательности $\left(\pi_{i}\right)_{i=1}^{\infty}$ такие, что $\pi_{i_{m}}\left(z_{l}\right) / \pi_{i_{m}}\left(z_{N}\right)<q$ для всех натуральньх $m$, то $\pi\left(z_{1}\right)=\cdots=\pi\left(z_{l}\right)=0$.

Д о к а з а т е ль с т в о. Докажем утверждение 1. Пусть $j \in$ $\{1, \ldots, N\}$ есть минимум из всех $k \in\{1, \ldots, N\}$ таких, что $\pi_{i}\left(z_{k}\right)>0$ для всех натуральных $i$. Очевидно, при любом натуральном $k<j$ имеем 
$\left(\pi_{1} \cdot \ldots \cdot \pi_{n}\right)\left(z_{k}\right)=0$ для всех достаточно больших $n$. Если $j=N$, то для всех достаточно больших $n$ получаем $\left(\pi_{1} \cdot \ldots \cdot \pi_{n}\right)\left(z_{k}\right)=1$, что завершает доказательство. Пусть $j \leqslant N-1$. Для каждого $k \in\{j, \ldots, N-1\}$ и каждого натурального $n$ пусть

$$
v_{k n}=\frac{\left(\pi_{1} \cdot \ldots \cdot \pi_{n}\right)\left(z_{k}\right)}{\left(\pi_{1} \cdot \ldots \cdot \pi_{n}\right)\left(z_{k+1}\right)}=\frac{\pi_{1}\left(z_{k}\right) \cdots \pi_{n}\left(z_{k}\right)}{\pi_{1}\left(z_{k+1}\right) \cdots \pi_{n}\left(z_{k+1}\right)} .
$$

По предположению для каждого $k \in\{j, \ldots, N-1\}$ имеем $v_{k n} \leqslant 1$, причем последовательность $\left(v_{k n}\right)_{n=1}^{\infty}$ не возрастает; поэтому $v_{k n} \leqslant 1$ для всех натуральных $n$. Следовательно, $\left(\pi_{1} \cdot \ldots \cdot \pi_{n}\right)\left(z_{k}\right) \leqslant\left(\pi_{1} \cdot \ldots \cdot \pi_{n}\right)\left(z_{k+1}\right)$ для всех $k \in\{j, \ldots, N-1\}$ и всех натуральных $n$. Для завершения доказательства достаточно показать, что последовательность $\left(\left(\pi_{1} \cdot \ldots \cdot\right.\right.$ $\left.\left.\pi_{n}\right)\left(z_{k}\right)\right)_{n=1}^{\infty}$ сходится при каждом $k \in\{j, \ldots, N-1\}$. Заметим, что для каждого $k \in\{j, \ldots, N-1\}$ невозрастающая последовательность $\left(v_{k n}\right)_{n=1}^{\infty}$ сходится. Покажем, что последовательность $\left(\left(\pi_{1} \cdot \cdots \cdot \pi_{n}\right)\left(z_{N}\right)\right)_{n=1}^{\infty}$ не убывает (здесь мы слегка модифицируем рассуждение, использованное выше при доказательстве леммы 2.1). По определению

$$
\left(\pi_{1} \cdot \pi_{2}\right)\left(z_{N}\right)=\frac{\pi_{1}\left(z_{N}\right) \pi_{2}\left(z_{N}\right)}{\sum_{k=1}^{N} \pi_{1}\left(z_{k}\right) \pi_{2}\left(z_{k}\right)}=\frac{\pi_{1}\left(z_{N}\right)}{\sum_{k=1}^{N-1} \pi_{1}\left(z_{k}\right) q\left(z_{k}\right)+\pi_{1}\left(z_{N}\right)},
$$

где $q\left(z_{k}\right)=\pi_{2}\left(z_{k}\right) / \pi_{2}\left(z_{N}\right)$ для $k \in\{1, \ldots, N-1\}$. Поскольку $q\left(z_{k}\right) \leqslant 1$ для $k \in\{1, \ldots, N-1\}$, имеем $\left(\pi_{1} \cdot \pi_{2}\right)\left(z_{N}\right) \geqslant \pi_{1}\left(z_{N}\right)$. Аналогично, рассуждая по индукции, устанавливаем, что $\left(\pi_{1} \cdot \ldots \cdot \pi_{n+1}\right)\left(z_{N}\right) \geqslant\left(\pi_{1} \cdot \ldots \cdot \pi_{n}\right)\left(z_{N}\right)$ для произвольного натурального $n$. Итак, последовательность $\left(\left(\pi_{1} \cdot \ldots \cdot\right.\right.$ $\left.\left.\pi_{n}\right)\left(z_{N}\right)\right)_{n=1}^{\infty}$ не убывает и, следовательно, сходится. Для всех натуральных $n$ имеем $\left(\pi_{1} \cdot \ldots \cdot \pi_{n}\right)\left(z_{N-1}\right)=v_{N-1, n}\left(\pi_{1} \cdot \ldots \cdot \pi_{n}\right)\left(z_{N}\right)$. Поскольку каждая из последовательностей $\left(v_{N-1, n}\right)_{n=1}^{\infty}$ и $\left(\left(\pi_{1} \cdot \ldots \cdot \pi_{n}\right)\left(z_{N}\right)\right)_{n=1}^{\infty}$ сходится, последовательность $\left(\left(\pi_{1} \cdot \ldots \cdot \pi_{n}\right)\left(z_{N-1}\right)\right)_{n=1}^{\infty}$ также сходится. Аналогично, рассуждая по индукции, устанавливаем, что последовательность $\left(\left(\pi_{1} \cdot \ldots \cdot \pi_{n}\right)\left(z_{k}\right)\right)_{n=1}^{\infty}$ сходится при каждом $k \in\{j, \ldots, N-1\}$. Утверждение 1 доказано.

Докажем утверждение 2. Пусть выполнены его предположения. Для всех достаточно больших натуральных $n$ имеем

$$
w_{n}=\frac{\left(\pi_{1} \cdot \ldots \cdot \pi_{n}\right)\left(z_{l}\right)}{\left(\pi_{1} \cdot \ldots \cdot \pi_{n}\right)\left(z_{N}\right)}=\frac{\pi_{1}\left(z_{l}\right) \cdots \pi_{n}\left(z_{l}\right)}{\pi_{1}\left(z_{N}\right) \cdots \pi_{n}\left(z_{N}\right)} \leqslant q^{s(n)},
$$

где $s(n)$ - максимум из всех $i_{m}(m \in\{1,2, \ldots\})$ таких, что $i_{m} \leqslant n$. Так как $s(n) \rightarrow \infty$ при $n \rightarrow \infty$, то $w_{n} \rightarrow 0$ при $n \rightarrow \infty$. Следовательно, $\pi\left(z_{l}\right)=\lim _{n \rightarrow \infty}\left(\pi_{1} \cdot \ldots \cdot \pi_{n}\right)\left(z_{l}\right)=0$. Поскольку $\pi\left(z_{1}\right) \leqslant \cdots \leqslant \pi\left(z_{l}\right)$, имеем $\pi\left(z_{1}\right)=\cdots=\pi\left(z_{l}\right)=0$. Утверждение 2 доказано.

Из теоремы 4.6, принимая во внимание теорему 4.4, сразу получаем, что для интеграционной сходимости последовательности вероят- 
ностей достаточно, чтобы все образующие ее вероятности, за исключением разве что их конечного числа, приоритизировали элементарные события в одном порядке.

Следствие 4.1. Пусть последовательность $\left(\pi_{i}\right)_{i=1}^{\infty}$ вероятностей из П непротиворечива и существует последовательность $\left(z_{k}\right)_{k=1}^{N}$ в $Z$ такая, что $\left\{z_{1}, \ldots, z_{N}\right\}=Z$ u $\pi_{i}\left(z_{1}\right) \leqslant \cdots \leqslant \pi_{i}\left(z_{N}\right)$ для всех достаточно больших натуральньх $i$. Тогда последовательность $\left(\pi_{i}\right)_{i=1}^{\infty}$ интеграчионно сходится и справедливо утверждение 2 теоремь 4.6.

4.2. Перестановки в интеграции последовательностей. Как отмечено выше (см. следствие 3.1), результат интеграции конечного числа вероятностей не зависит от порядка, в котором производится интеграция. Отсюда сразу вытекает, что конечные перестановки в бесконечных последовательностях вероятностей не меняют их частичных пределов. Именно, справедливо следующее.

Следствие 4.2. Пусть последовательность $\left(\pi_{i}\right)_{i=1}^{\infty}$ вероятностей из П непротиворечива, $k$ - натуральное, $\left(i_{1}, i_{2}, \ldots, i_{k}\right)-$ перестановка в наборе $(1,2, \ldots, k)$ и

$$
\begin{cases}\pi_{i_{j}}, & \text { если } j \in\{1, \ldots, k\}, \\ \pi_{j}, & \text { если } j \in\{k+1, k+2, \ldots\} .\end{cases}
$$

Тогда множества всех частичных интеграционных пределов последовательностей $\left(\pi_{i}\right)_{i=1}^{\infty} u\left(\pi_{i}^{*}\right)_{i=1}^{\infty}$ совпадают.

Следующий пример показывает, что это утверждение не распространяется на бесконечные перестановки в последовательностях вероятностей.

П р и м е р 4.3. Пусть $Z=\left\{z_{1}, z_{2}\right\}$ и вероятности $\pi_{i}^{(1)}, \pi_{i}^{(2)} \in \Pi$ $(i \in\{1,2, \ldots\})$ таковы, что

$$
\pi_{i}^{(1)}\left(z_{1}\right)=1-\varepsilon_{i}, \quad \pi_{i}^{(1)}\left(z_{2}\right)=\varepsilon_{i}, \quad \pi_{i}^{(2)}\left(z_{1}\right)=\varepsilon_{i}, \quad \pi_{i}^{(2)}\left(z_{2}\right)=1-\varepsilon_{i},
$$

где $\varepsilon_{i} \in(0,1)$ и $\lim _{i \rightarrow \infty} \varepsilon_{i}=0$. Для произвольных $i$ и $j \geqslant i$ обозначим

$$
\pi_{i j}^{(1)}=\pi_{i}^{(1)} \cdot \ldots \cdot \pi_{j}^{(1)}, \quad \pi_{i j}^{(2)}=\pi_{i}^{(2)} \cdot \ldots \cdot \pi_{j}^{(2)} .
$$

По теореме 4.3

$$
\pi_{i j}^{(1)} \rightarrow \pi^{(1)} \quad \text { в } \Pi, \quad \pi_{i j}^{(2)} \rightarrow \pi^{(2)} \quad \text { в } \Pi,
$$

где $\pi^{(1)}$ и $\pi^{(2)}$ сосредоточены в $z_{1}$ и в $z_{2}$ соответственно. Возьмем натуральные $k_{1}, k_{2}, \ldots$ и положим

$$
\begin{aligned}
\pi_{j}^{\prime} & =\pi_{j}^{(1)} \quad\left(j \in\left\{1, \ldots, k_{1}\right\}\right), \\
\pi_{k_{1}+1}^{\prime} & =\pi_{1}^{(2)}
\end{aligned}
$$




$$
\begin{aligned}
\pi_{j}^{\prime}= & \pi_{j}^{(1)} \quad\left(j \in\left\{k_{1}+2, \ldots, k_{1}+2+k_{2}\right\}\right), \\
\pi_{k_{1}+k_{2}+3}^{\prime}= & \pi_{2}^{(2)}, \\
& \left.\left.\ldots \ldots \ldots \ldots \ldots \ldots \ldots \ldots, \ldots \ldots, m_{s+1}\right\}\right), \\
\pi_{j}^{\prime}= & \pi_{j}^{(1)}\left(j \in \left\{m_{s}, \ldots \ldots,\right.\right. \\
\pi_{m_{s+1}+1}^{\prime}= & \pi_{s+1}^{(2)}
\end{aligned}
$$

где $m_{s}=\sum_{l=1}^{s} k_{l}+s+1$. Очевидно, что последовательность $\left(\pi_{j}^{\prime}\right)_{j=1}^{\infty}$ непротиворечива. Возьмем положительные $\delta_{1}, \delta_{2}, \ldots$ такие, что $\lim _{s \rightarrow \infty} \delta_{s}=0$. Принимая во внимание $(4.7)$, тот факт, что $\pi^{(1)}$ есть нуль относительно интеграции как умножения, и непрерывность интеграции, подберем последовательно натуральные $k_{1}, k_{2}, \ldots$ так, что

$$
\begin{aligned}
\left(\pi_{1}^{\prime} \cdot \ldots \cdot \pi_{m_{s+1}+1}^{\prime}\right)\left(z_{1}\right) & =\left(\pi_{1}^{\prime} \cdot \ldots \cdot \pi_{m_{s}+1}^{\prime} \cdots \pi_{m_{s}+1 m_{s}+1}^{(1)} \cdot \pi_{m_{s+1}+1}^{(2)}\right)\left(z_{1}\right) \\
& =\left(\pi_{m_{s}+1 m_{s+1}}^{(1)} \cdot\left(\pi_{1}^{\prime} \cdot \ldots \cdot \pi_{m_{s}+1}^{\prime} \cdot \pi_{m_{s+1}+1}^{(2)}\right)\right)\left(z_{1}\right) \\
& >1-\delta_{s} .
\end{aligned}
$$

Тогда на основании теоремы 2.1 для всех $k \in\left\{m_{s+1}+2, \ldots, m_{s+3}\right\}=$ $\left\{m_{s+2}, \ldots, m_{s+3}\right\}$ получаем

$$
\begin{aligned}
\left(\pi_{1}^{\prime} \cdot \ldots \cdot \pi_{k}^{\prime}\right)\left(z_{1}\right) & =\left(\left(\pi_{1}^{\prime} \cdot \ldots \cdot \pi_{m_{s+1}+1}^{\prime}\right) \cdot \pi_{m_{s+2}} \cdot \ldots \cdot \pi_{k}^{\prime}\right)\left(z_{1}\right) \\
& =\left(\left(\pi_{1}^{\prime} \cdot \ldots \cdot \pi_{m_{s+1}+1}^{\prime}\right) \cdot \pi_{m_{s+2}}^{(1)} \cdot \ldots \cdot \pi_{k}^{(1)}\right)\left(z_{1}\right) \\
& >\left(\pi_{1}^{\prime} \cdot \ldots \cdot \pi_{m_{s+1}+1}^{\prime}\right)\left(z_{1}\right)>1-\delta_{s} .
\end{aligned}
$$

Таким образом, $\pi^{(1)}$ есть интеграционный предел последовательности $\left(\pi_{j}^{\prime}\right)_{j=1}^{\infty}$. Теперь зададим вероятности $\pi_{1}^{\prime \prime}, \pi_{2}^{\prime \prime}, \ldots \in \Pi$, меняя местами $\pi_{j}^{(1)}$ и $\pi_{j}^{(2)}$ в определении $\pi_{1}^{\prime}, \pi_{2}^{\prime}, \ldots$. Рассуждая как выше, устанавливаем, что $\pi^{(2)}$ есть интеграционный предел последовательности $\left(\pi_{j}^{\prime \prime}\right)_{j=1}^{\infty}$. Ясно, что последовательность $\left(\pi_{j}^{\prime \prime}\right)_{j=1}^{\infty}$ получается их последовательности $\left(\pi_{j}^{\prime}\right)_{j=1}^{\infty}$ бесконечной перестановкой. Таким образом, бесконечная перестановка в последовательности $\left(\pi_{j}^{\prime}\right)_{j=1}^{\infty}$, оставляя ее интеграционно сходящейся, меняет ее интеграционный предел.

Заключение. Настоящая работа мотивирована вопросами, возникающими в прикладных исследованиях при анализе неточных данных, доставляемых альтернативными независимыми источниками. Предложен метод интеграции данных, представленных в виде вероятностных распределений. Проведено начальное исследование метода ограниченное простейшим случаем конечного множества элементарных событий. Среди вопросов, запланированных к дальнейшему изучению, отметим следующие: распространение обозначенной теории на случаи бесконечных вероятностных пространств, в частности пространств с вероятностями, заданными плотностями распределения; построение «таблицы 
умножения» стандартных распределений — результатов их попарной интеграции; сравнение информативности априорных вероятностей и результатов их апостериорной интеграции в терминах различных мер сосредоточенности; распространение предложенного метода на случайные процессы; вопросы оптимального выбора априорных вероятностей для апостериорной интеграции.

Непосредственным импульсом для начала автором этой работы послужили многочисленные обсуждения задачи об интеграции вероятностных оценок первичной продукции лесных экосистем, инициированные А. З. Швиденко; автор выражает ему искреннюю благодарность. Автор благодарен Е.А. Ровенской, ознакомившей его с основной литературой по эмпирическим методам интеграции распределений, применяемым в исследованиях по климатическим изменениям. Автор признателен А.Н. Ширяеву, а также участникам Международной школы молодых ученых «Современные проблемы математики и ее приложений» (Екатеринбург, 2-8 февраля 2014 г.) за обсуждения отдельных частей работы.

\section{СПИСОК ЛИТЕРАТУРЫ}

1. Ширяев А. Н. Вероятность-1. М.: МЦНМО, 2011, 551 с.

2. Blackwell D. Comparisons of experiments. - Proceedings of the Second Berkeley Symposium on Mathematical Statistics and Probability. Berkeley-Los Angeles: Univ. of California Press, 1951, p. 93-102.

3. Blackwell D. Equivalent comparisons of experiments. - Ann. Math. Statist., 1953, v. 24 , № 2 , p. 265-272.

4. Frühwirth-Schnatte S. Finite Mixture and Markov Switching Models. New York: Springer, 2006, $492 \mathrm{p}$.

5. Knutti R., Furrer R., Tebaldi C., Cermak J., Meehl G.A. Challenges in combining projections from multiple climate models. - J. Climate, 2010, v. 23, № 10, p. 27392758.

6. Nilsson S., Shvidenko A., Jonas M., McCallum I., Thompson A., Balzter H. Uncertainties of a regional terrestrial biota full carbon account: A systems analysis. Accounting for Climate Change. Uncertainty in Greenhouse Gas Inventorios Verification, Compliance, and Trading. Dordrecht: Springer, 2007, p. 5-21.

7. Rajagopalan B., Lall U., Zebiak S.E. Categorical climate forecasts through regularization and optimal combination of multiple GCM ensembles. - Monthly Weather Review, 2002, v. 130, № 7, p. 1792-1811.

8. Robertson A. W., Lall U., Zebiak S. E., Goddard L. Improved combination of multiple atmospheric GCM ensembles for seasonal prediction. - Monthly Weather Review, 2004 , v. 132 , № 12 , p. $2732-2744$.

9. Sulganik E., Zilcha I. The value of information: The case of signal-dependent opportunity sets. - J. Economic. Dynam. Control, 1997, v. 21, № 10, p. 1615-1625.

10. Tebaldi $C$., Knullti $R$. The use of the multi-model ensemble in probabilistic climate projections. - Philos. Trans. Roy. Soc. Lond. Ser. A, 2007, v. 365, № 1857, p. 20532075.

11. Wald A. Statistical decision functions. - Ann. Math. Statist., 1949, v. 20, № 2, p. 165-205. 